\title{
CONDUCTIVE-RADIATIVE HEAT TRANSFER IN GREY MATERIALS
}

\author{
BY \\ MIKA LAITINEN (Numerola Oy, Inc., Väinönkatu 11A, FIN-40100 Jyväskylä, Finland)
}

AND

TIMO TIIHONEN (Department of Mathematical Information Technology, University of Jyväskylä, P.O. Box 35, FIN-40351 Jyväskylä, Finland)

\begin{abstract}
We study the well-posedness of a class of models describing heat transfer by conduction and radiation. For that purpose we propose an abstract mathematical framework that allows us to prove existence, uniqueness and the comparison principle for the weak solution with minimal or almost minimal a priori assumptions for the data. The theory covers different types of grey materials, that is, both semitransparent and opaque bodies as well as isotropic or nonisotropic scattering/reflection provided that the material properties do not depend on the wavelength of the radiation. To demonstrate the use of the abstract theory we consider in detail two examples, heat transfer between opaque bodies with diffuse-grey surfaces and a model with semitransparent material and specularly reflecting surfaces.
\end{abstract}

1. Introduction. Heat radiation is a significant factor in heat transfer in many cases, in particular, when hot bodies are separated by a transparent or semitransparent medium with relatively low heat conductivity. In fact, these conditions are often already met at room temperatures - why else would we speak of radiators?

Although the basic physical principles of heat radiation are well understood and there exists a number of engineering textbooks where a whole hierarchy of different radiation models are used for practical computations (see, for example, [2], [13], [18], [19]) the coupling of heat radiation to other heat transfer mechanisms has received surprisingly little mathematical attention. Concerning the simplest nontrivial case of a conductive body with nonconvex opaque radiating surface, we are aware of three independent works: [15], [16], and our previous work starting from [20]. They all study some properties of the operators related to radiative transfer and show the existence of a weak solution under some restrictions (no enclosed surfaces, limitations to material properties). The basic case has been extended to cover several conductive bodies [17], [21] and time-dependent

Received November 16, 1999.

2000 Mathematics Subject Classification. Primary 35J65, 45R05, 80A20.

Key words and phrases. Heat radiation, Stefan-Boltzmann law, nonlocal problems.

(C)2001 Brown University 


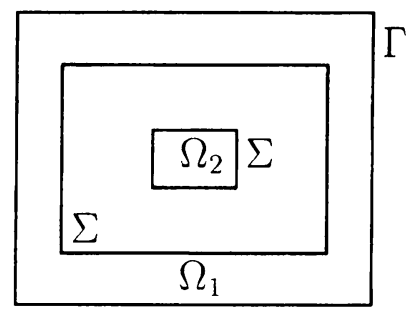

FIG. 1. Conductive-radiative heat transfer between two disjoint bodies

problems [12], [21]. In the case of semitransparent media, the analysis has been carried out in the one-dimensional case with nonreflecting surfaces [9] and in two and three dimensions with diffusively reflecting surfaces [11].

The mathematical structures discovered independently in the above-mentioned papers are similar. Therefore, one goal of the present study was to find a general framework, which includes all of the above cases as well as some models that have not been analysed before. This framework consists of a set of abstract structural assumptions that allow us to prove existence and uniqueness for a weak solution in both the stationary and the time-dependent case. In our opinion, the general theory is important not only for its own sake but also because the generic theory is needed if one wants to answer on a rational basis questions such as which simplified models can be used in a particular situation or which properties of the model should be conserved when designing numerical approximation methods. Or to put it shortly but provocatively, how to change the "art" of numerical heat radiation to a science.

We do not consider the most general setting however. We shall restrict ourselves to grey materials, that is, the radiative coefficients of these materials do not depend on a wavelength. Also, temperature dependent material properties are beyond the scope of this paper. The results presented in this work could be generalized to some cases involving materials with wavelength or temperature dependent radiative properties, but since this part of the theory has not yet reached full generality, we postpone its treatment to later works. It seems that the mathematical analysis of non-grey models is entirely an open field.

In generalizing the theory, we had particulary the following problem in mind. This problem can be considered as an abstraction of contactless heat transfer in a protected environment arising, for example, in semiconductor applications and will be examined in detail in Sec. 3. Let $\Omega=\Omega_{1} \cup \Omega_{2} \subset \mathbb{R}^{3}$ denote a union of two disjoint, conductive and opaque bodies surrounded by a transparent and non-conductive medium; see Fig. 1. We assume that the radiative surfaces $\Sigma$ and $\Gamma$ are diffuse and grey, that is, the emissivity coefficient $\epsilon$ of these surfaces depends neither on direction nor on wavelength of the radiation. Now, the stationary heat equation for absolute temperature $T$ reads as

$$
\begin{aligned}
-\nabla \cdot(k \nabla T) & =f \quad \text { in } \Omega_{1} \cup \Omega_{2}, \\
-k \frac{\partial T}{\partial n} & =q_{\mathrm{r}} \quad \text { on } \Sigma \cup \Gamma,
\end{aligned}
$$


where $n$ is the outward unit normal, $k$ is the coefficient of heat conduction, $f$ is a given heat source, and $q_{\mathrm{r}}$ is the radiative heat flux, that is, the difference between incoming radiation $u$ and outgoing radiation $\rho$,

$$
q_{\mathrm{r}}(x)=\rho(x)-u(x) .
$$

For convex $\Gamma$ we can assume the external radiation to be known data. Therefore, the radiative flux on $\Gamma$ consists only of the local emission given by the Stefan-Boltzmann law, $\left.q_{\mathrm{r}}\right|_{\Gamma}=\epsilon \sigma\left(T^{4}-T_{0}^{4}\right)$. Here $\sigma$ denotes the Stefan-Boltzmann constant and $T_{0}^{4}$ is the effective external radiation temperature. The surface $\Sigma$, however, receives radiation from other parts of itself, leading to a relation

$$
u(x)=(K \rho)(x), \quad \text { on } \Sigma,
$$

where $K$ is an integral operator with kernel defined on $\Sigma \times \Sigma$. Finally, we note that the outgoing radiation $\rho$ on $\Sigma$ is a combination of emission and reflected fraction of incoming radiation,

$$
\rho=\epsilon \sigma T^{4}(x)+(1-\epsilon) u(x)=\epsilon \sigma T^{4}(x)+(1-\epsilon)(K \rho)(x) .
$$

Solving, for the time being formally, $\rho$ as a function of $T$ we can write our problem in variational form as

$$
\int_{\Omega} k \nabla T \nabla \varphi d x \int_{\Sigma}\left(G \sigma T^{4}\right) \varphi d s+\int_{\Gamma} \epsilon \sigma T^{4} \varphi d s=\int_{\Omega} f \varphi d x+\int_{\Gamma} \epsilon \sigma T_{0}^{4} \varphi d s,
$$

where the operator $G$ is defined as $G v:=(I-K)(I-(1-\epsilon) K)^{-1} \epsilon v$.

The following aspects complicate the analysis of the above model: First, the radiation terms on $\partial \Omega$ are well defined only if $T$ and $\varphi$ are in $L^{5}(\partial \Omega)$ and, thus, the weak solution should be sought from the space $H^{1}(\Omega) \cap L^{5}(\partial \Omega)$. Second, coercivity is difficult to obtain because $\Omega$ is disconnected: the sole heat conduction part is not coercive in $H^{1}(\Omega)$ and also the nonlocal radiation part on $\Sigma$ lacks coercivity in $L^{5}(\Sigma)$ since $G$ maps constants to zero (as one expects no isothermal heat flux). In fact, previously [12], [21], [20] the coercivity of the coupled problem has been obtained only by assuming that the radiative surface $\Sigma$ is not an enclosure. In this work our aim is to remove such restrictions.

When generalizing the above model we recognize the following steps. First, in each specific case, we solve the equations governing radiative transport for a given temperature field, which leads us to define the linear radiation operator termed $G$. If the radiative system contains also semitransparent materials, the operator $G$ is defined over semitransparent volumes and opaque surfaces. Thus, in the weak formulation of the abstract problem, we integrate the term $G \sigma T^{4}$ with respect to a more general measure $\mu$, measuring both volumes and surfaces. The operator $G$ is then understood as a mapping from the space $L_{\mu}^{p}$ to itself $(1 \leq p \leq \infty)$. Finally, we postulate the general properties of the operator $G$, to be verified in each specific case. In determining these properties, a coercivity result, which somewhat resembles the inequalities of Poincaré and Friedrichs, plays an essential role. The main tools are certain compactness properties of radiation operators, interpolation theory of operators and theory of positive operators.

This paper is organized as follows: In Sec. 2 we describe the abstract framework and state our main results concerning the general conductive-radiative problem. In Sec. 3 we analyse examples of typical conductive-radiative models and show that they fit into 
the abstract framework. First, we consider the example described above (diffuse-grey surfaces) without any unphysical restrictions on surface cmissivity. Secondly, we analyse heat transfer in semitransparent materials with specularly reflecting surfaces. The rest of the paper is devoted to the analysis of the abstract model. In Sec. 4 we prove the existence of solutions in the stationary case by showing that our problem is pseudomonotone and coercive. In Sec. 5 we prove the uniqueness via the comparison principle using an idea borrowed from the analysis of nonlinear heat conduction [10]. Section 6 provides $L^{\infty}$ estimates in the stationary case utilizing the Moser iteration technique. The existence and uniqueness as well as the comparison principle for the time-dependent case are proved in Sec. 7.

Notation. For a Borel measure $\mu$, we denote the duality between $L_{\mu}^{p}$ and $L_{\mu}^{q}$ by

$$
\langle u, v\rangle_{\mu}:=\int_{\Lambda \cup \Sigma} u v d \mu, \quad u \in L_{\mu}^{p} \text { and } v \in L_{\mu}^{q},
$$

when $1 \leq p \leq \infty$ and $p$ and $q$ are conjugate exponents, that is, $1 / p+1 / q=1$. For an operator $H$ that maps $L_{\mu}^{p}$ to itself for every $1 \leq p \leq \infty$, we extend the common notion and say that $H$ is symmetric if $\langle H u, v\rangle_{\mu}=\langle u, H v\rangle_{\mu}$ for all $u \in L_{\mu}^{p}$ and $v \in L_{\mu}^{q}$ and for the pair of conjugate exponents $1 \leq p, q \leq \infty$. Further, we call an operator $H$ positive if $u \geq 0$ implies $H u \geq 0$, and denote the positive and negative parts of a function by either sub- or superscript: $u^{+}=u_{+}=\max (u, 0)$ and $u^{-}=u_{-}=\max (-u, 0)$. Finally, we denote by $C>0$ different positive constants appearing in the proofs.

2. Abstract framework. In this section we introduce an abstract formulation for a family of conductive-radiative models and state our main results. Some concrete examples of models that are covered by the abstract theory will be discussed later in Sec. 3.

We begin with conduction. Let $\Omega=\Omega_{1} \cup \Omega_{2} \cup \cdots \cup \Omega_{k} \subset \mathbb{R}^{n}(n=2.3)$ denote the union of open, bounded, connected and disjoint sets containing the conductive parts of the system. The boundaries $\partial \Omega_{k}$ are assumed Lipschitz as well as other surfaces in this work. We denote by $\Gamma$ a subset of $\partial \Omega$ where local heat transfer (i.e., "radiation to infinity" or convective heat transfer) occurs and define an operator $A$ through

$$
\langle A u, v\rangle:=\int_{\Omega} a_{i j} \partial_{i} u \partial_{j} v d x+\int_{\Gamma} \zeta|u|^{p-1} u v d s,
$$

where the summation convention is understood, $p>0$, and the coefficients $a_{i j}$ as well as $\zeta \geq 0$ are bounded. The natural domain for the operator $A$ is

$$
H^{1}(\Omega) \cap L_{\gamma}^{p+1}(\Gamma) .
$$

where the measure $\gamma$ is the surface measure of $\Gamma$ weighted with the coefficient $\zeta$, i.e., $\gamma(B)=\int_{\Gamma \cap P} \zeta(s) d s$ for a Borel set $B \subset \mathbb{R}^{n}$. The null space of $A$ is denoted by

$$
\mathcal{N}(A)=\left\{u \in H^{1}(\Omega) \cap L_{\gamma}^{p+1}(\Gamma): A u=0\right\} .
$$

We assume that $\left\{a_{i j}\right\}$ is strictly elliptic, that is,

(H1) There exists a constant $C>0$ such that

$$
\langle A u, u\rangle \geq C \int_{\Omega}|\nabla u|^{2} d x \quad \text { for all } u \in H^{1}(\Omega) .
$$


The domain of the nonlocal radiation models is contained in the sets $\Lambda$ and $\Sigma . \Lambda \subset \Omega$ denotes an open set containing the material that is semitransparent to radiation whereas $\Sigma$ denotes the union of opaque and oriented surfaces where nonlocal surface radiation occurs (thus $\Sigma \cap \Gamma=\varnothing$ ). Note that neither $\Lambda$ nor $\Sigma$ are in general connected. The optical activity in $\Lambda \cup \Sigma$ is characterized by a nonzero measure $\mu$,

$$
\mu(B)=\int_{\Lambda \cap B} w_{\Lambda}(x) d x+\int_{\Sigma \cap B} w_{\Sigma}(s) d s, \quad \text { for every Borel set } B \subset \mathbb{R}^{n},
$$

where $w_{\Lambda} \in L^{\infty}(\Lambda)$ and $w_{\Sigma} \in L^{\infty}(\Sigma)$ are nonnegative. The radiative heat exchange in $\Lambda \cup \Sigma$ is represented by a nonlocal operator $G$, which is assumed to satisfy:

(H2) $G$ is a linear operator from $L_{\mu}^{p}$ to itself for $1 \leq p \leq \infty$.

(H3) Both $G$ and its adjoint $G^{*}$ satisfy $G 1 \geq 0$ and $G^{*} 1 \geq 0$.

(H4) Both $H:=I-G$ and $H^{*}$ are positive.

(H5) $G$ can be written also as $G=E-F$ where $E$ is a multiplication operator, $(E u)(x)=e(x) u(x)$ with $0<e_{0} \leq e(x) \leq 1$, and $F$ is a compact operator in $L_{\mu}^{p}$ for $1<p<\infty$.

Further, we define the null space of $G$ as

$$
\mathcal{N}(G)=\left\{u \in L_{\mu}^{1}: G u=0\right\}
$$

and assume that

(H6) $\mathcal{N}(A) \cap \mathcal{N}(G)=\{0\}$.

(H7) For $q>0,|u|^{q} u \in \mathcal{N}(G)$ implies $u \in \mathcal{N}(G)$.

The condition (H3) means that in the isothermal case either radiative heat flux is zero $(G 1=0)$ or part of the radiation is escaping the system $(G 1>0)$. The conditions (H6) and (H7) ensure the coercivity by linking the disjoint conducting and radiating components to each other and (H7) basically says that the null space of $G$ consists of piecewise constants.

In this work we consider the following two problems. First, in a stationary case our problem reads as: Given $f \in V^{*}$, find $u \in V$ such that

$$
\langle Q u, v\rangle:=\langle A u, v\rangle+\int_{\Lambda \cup \Sigma}\left(G|u|^{3} u\right) v d \mu=\langle f, v\rangle \quad \forall v \in V,
$$

where the solution space $V$ is given by

$$
V=H^{1}(\Omega) \cap L_{\mu}^{5}(\Lambda \cup \Sigma) \cap L_{\gamma}^{p+1}(\Gamma) .
$$

We note that the space $V$ is reflexive by the arguments given in [4]. Observe also that since the Stefan-Boltzmann law is physical only for nonnegative values of temperature, we can replace the term $u^{4}$ by $|u|^{3} u$ for mathematical convenience.

Secondly, we consider the time-dependent counterpart of (2.2): For $f \in X^{*}$ and $u_{0} \in L^{2}(\Omega)$, we seek $u \in W$ such that

$$
\begin{aligned}
\left\langle u^{\prime}(t), v\right\rangle_{V}+\langle Q u(t), v\rangle_{V} & =\langle f(t), v\rangle_{V}, \\
u(0) & =u_{0},
\end{aligned}
$$

for all $v \in V$ and almost all $t \in[0, T]$. Here the operator $Q$ is defined as in (2.2). Note that to simplify the presentation we assume $p=1$ on $\Gamma$ in the time-dependent case. The 
spaces $X$ and $W$ are defined as

$$
\begin{aligned}
X & =L^{2}\left(0 . T ; H^{1}(\Omega)\right) \cap L^{5}\left(0, T: L_{\mu}^{5}\right) . \\
W & =\left\{u: u \in X, u^{\prime} \in X^{*}\right\} .
\end{aligned}
$$

Finally, our main results can be crystallized as:

THEOREM 1. Under the above hypotheses, the following hold:

(i) There exists a unique solution to (2.2).

(ii) If $u_{0} \in L^{5}(\Omega)$ and $f(t)$ satisfies

$$
f(t) \in L^{2}((0 . T) \times \Omega)+L^{5+\delta}\left(0 . T: L^{5 / 3+\delta}(\Sigma \cup \Gamma)\right)
$$

for some $\delta>0$, then there exists a unique solution for (2.3)--(2.4).

(iii) Both problems (2.2) and (2.3)-(2.4) satisfy a comparison principle (as specified in Sections 5 and 7$)$.

(iv) If $f \in L^{q}(\Omega)+L^{q}(\Sigma \cup \partial \Omega)$ for some $q>2$, then the solution of $(2.2)$ is in $L^{\infty}(\Omega) \cap L^{\infty}(\Sigma \cup \partial \Omega)$.

REMARK 2.1. If temperature is prescribed on a part of $\partial \Omega$, then the results in Sections 4-6 are valid also in this case (of course, the definition of the function spaces must then be changed). However, to keep the presentation simple, we do not discuss this aspect in this work.

3. Physical models. In this section we illustrate the abstract theory with concrete examples. First, we recall and extend the models with diffuse-grey surfaces studied in $[12],[15],[17]$ as well as in our earlier works [21], [20]. Secondly, we study heat transfer in a semitransparent material with specular boundary reflections. The model with specular reflections is challenging since it requires the directional distribution of radiation to be taken into account. Let us also mention that our analysis of semitransparent material with diffuse boundary reflections [11] offers another example which fits into our framework. In the final subsection we discuss models with several conducting and radiating components. For a more detailed description of the physics we refer to [18], [19], [13], and [2].

3.1. Radiation on diffuse-grey surfaces. We investigate next the problem posed in the introduction; see Fig. 1. Our conductive-radiative system $\Omega=\Omega_{1} \cup \Omega_{2} \subset \mathbb{R}^{3}$ consists of two conducting and opaque bodies that are in contact by radiation through a transparent medium bounded by a diffuse-grey surface $\Sigma$. The surface $\Sigma$ is assumed to be an enclosure (i.e., $\mathbb{R}^{3} \backslash \Sigma$ is nonconnected). We recall from the introduction the heat equation for absolute temperature $T$,

$$
\begin{aligned}
-\nabla \cdot(k \nabla T) & =f & & \text { in } \Omega, \\
-k \frac{\partial T}{\partial n} & =\epsilon \sigma\left(T^{4}-T_{0}^{4}\right) & & \text { on } \Gamma, \\
-k \frac{\partial T}{\partial n} & =q_{\mathrm{r}}=\rho-u & & \text { on } \Sigma,
\end{aligned}
$$


with the incoming radiation $\rho$ and the outgoing radiation $u$ related by

$$
u=K \rho, \quad \rho=\epsilon \sigma T^{4}+(1-\epsilon) u=\epsilon \sigma T^{4}+(1-\epsilon) K \rho .
$$

Note that the physical values of the emissivity $\epsilon=\epsilon(x)$ are limited by $0 \leq \epsilon(x) \leq 1$. The integral operator $K$ can be derived as follows: We denote by $S$ the set of all directions, that is, surface of a unit sphere in $\mathbb{R}^{3}$ and by $d=d(x, \omega)$ the distance of $x \in \Sigma$ from the surface $\Sigma$ itself in the direction of $-\omega$. Then, the incoming radiation $u(x)$ consists simply of the outgoing radiation from every other point of $\Sigma$ that reaches $x$ :

$$
u(x)=\int_{S} \rho(x-d \omega)(\omega \cdot n(x))^{+} d \omega=:(K \rho)(x) .
$$

This can also be written in Cartesian coordinates by introducing the change of variables $y=x-d \omega, \omega=(x-y) /|x-y|, d \omega=n(y) \cdot(x-y)|x-y|^{-3} d y$,

$$
(K \rho)(x)=\int_{\Sigma} \frac{n(y) \cdot(x-y) n(x) \cdot(y-x)}{\pi|x-y|^{4}} \Xi(x, y) \rho(y) d y,
$$

where $n$ is the outward normal and $\Xi$ is the visibility factor:

$$
\Xi(x, y)= \begin{cases}1 & \text { if } x \text { and } y \text { see each other, } \\ 0 & \text { otherwise. }\end{cases}
$$

Here the statement " $x$ and $y$ see each other" means that there is no opaque material between $x$ and $y$ (i.e., $\overline{x y} \cap \Omega=\varnothing$ in this example).

LEmma 1 . Let $1 \leq p \leq \infty$. Then the operator $K$ maps $L^{p}(\Sigma)$ to itself and

(i) $K$ is symmetric,

(ii) $K$ is positive and compact,

(iii) $K 1=1$ and $\|K\|_{L^{p}(\Sigma)}=1$,

(iv) the eigenvalue 1 of $K$ is simple.

Proof. For the proof of (i)-(iii) see [20]. To prove (iv), we note that if there is a set $M \subset \Sigma$ of positive surface measure such that $u>0$ in $M$ and $x$ sees $M$, then $(K u)(x)>0$. Hence, there is an integer $N$ such that the operator $K^{N}$ has strictly positive kernel. Consequently, the eigenvalue 1 is simple according to the Doob-KreinRutman Theorem [3].

To resolve $\rho$ from (3.4), we have to show that the operator $I-(1-\epsilon) K$ is invertible in $L^{5 / 4}(\Sigma)$. In our previous works [20], [21] and [11] this has been obtained assuming $\epsilon \geq \epsilon_{0}>0$. To show that also the materials with arbitrary low emissivities can be treated, we assume next only that $\epsilon$ is nonnegative.

Lemma 2. Let $1 \leq p \leq \infty$ and $0 \leq \epsilon(x) \leq 1, \epsilon \neq 0$. Then the operator $I-(1-\epsilon) K$ from $L^{p}(\Sigma)$ to itself is invertible and this inverse is positive.

Proof. We shall prove that the spectral radius of the operator $(1-\epsilon) K$ is strictly less than one. Then the inverse exists and can be written as the Neumann series

$$
(I-(1-\epsilon) K)^{-1}=\sum_{i=0}^{\infty}((1-\epsilon) K)^{i}
$$

which shows that the inverse is positive. 
Since $(1-\epsilon) K$ is compact. it suffices to prove that 1 is not an eigenvalue of $(1-\epsilon) K$. Thus, suppose there is $u \in L^{p}(\Sigma)$ such that $(1-\epsilon) K u=u$. Then, since $(1-\epsilon) K$ is a positive and compact operator, we have $u \geq 0$, and hence

$$
\int_{\Sigma} u d s=\int_{\Sigma}(1-\epsilon) K u d s=\int_{\Sigma} u d s-\int_{\Sigma} \epsilon K u d s
$$

according to (ii) and (iii). Therefore, $\epsilon K u=0$ a.e. on $\Sigma$ and hence $K u=u$. Now, from (iv) it follows that $u$ is a constant, but since $(1-\epsilon) K u=u$, this constant has to be zero.

Now, we are ready to formulate the problem (3.1)-(3.4) in the abstract form. First, assuming $T \in L^{5}(\Sigma)$, and solving $\rho$ from (3.4), we have

$$
q_{\mathrm{r}}=(I-K) \rho=(I-K)(I-(1-\epsilon) K)^{-1} \epsilon \sigma T^{4}:=G \sigma T^{4},
$$

or, alternatively, by rearranging the terms in (3.4),

$$
q_{\mathrm{r}}=\epsilon \sigma T^{4}-\epsilon u=\epsilon \sigma T^{4}-\epsilon K(I-(1-\epsilon) K)^{-1} \epsilon \sigma T^{4},
$$

which physically means that $q_{\mathrm{r}}$ is composed of the difference between emitted and absorbed radiation. Then, defining the mapping $A$ from $H^{1}(\Omega) \cap L_{\gamma}^{5}$ to $\left(H^{1}(\Omega) \cap L_{\gamma}^{5}\right)^{*}$ by

$$
\langle A T, \varphi\rangle=\int_{\Omega} k \nabla T \cdot \nabla \varphi d x+\int_{\Gamma} \epsilon \sigma|T|^{3} T \varphi d s
$$

and setting $d \mu=\sigma d s$, we can write our problem in a weak form as

$$
\langle A T, \varphi\rangle+\int_{\Sigma} G|T|^{3} T \varphi d \mu=\langle\tilde{f}, \varphi\rangle \quad \forall \varphi \in V:=H^{1}(\Omega) \cap L_{\mu}^{5} \cap L_{\gamma}^{5},
$$

where $\tilde{f}$ now contains also the data term on $\Gamma$. We examined this formulation already in [20], [21], and proved that the operator $H:=I-G$ is symmetric, positive and satisfies $H 1=1$. However, the disadvantage of this formulation is that now $E u=\epsilon(x) u$ and $F u=\epsilon K(I-(1-\epsilon) K)^{-1} \epsilon u$ so that the assumption (H5) holds only if $\epsilon \geq \epsilon_{0}>0$. Fortunately, other alternatives to choose $\mu$ and $G$ exist.

The trick is to formulate the problem a bit differently by defining a measure $\tilde{\mu}$ such that $d \tilde{\mu}=\epsilon \sigma d s$. Then, setting

$$
\widetilde{G}=I-\widetilde{H}=I-K(I-(1-\epsilon) K)^{-1} \epsilon,
$$

we have

$$
\int_{\Sigma} G|T|^{3} T \varphi d \mu=\int_{\Sigma} \tilde{G}|T|^{3} T \varphi d \tilde{\mu} .
$$

Morcover, the properties (H2)-(H5) for $\widetilde{G}$ can easily be deduced from the corresponding properties of $G$, without assuming $\epsilon$ strictly positive.

It remains to verify the hypotheses (H6) and (H7). Now, the space $\mathcal{N}(A)$ consists of functions that are constant in $\Omega_{2}$ and zero in $\Omega_{1}$ provided $k$ and $\zeta=\left.\epsilon\right|_{\Gamma}$ are chosen properly. Therefore, if we can prove that 1 is a simple eigenvalue of $\widetilde{H}$, we are done.

Lemma 3. Let $0 \leq \epsilon(x) \leq 1$ and $\epsilon \neq 0$. Then 1 is a simple cigenvalue of the operator $\widetilde{H}: L_{\tilde{\mu}}^{1} \mapsto L_{\tilde{\mu}}^{1}$. 
Proof. We shall prove that $\widetilde{H}^{N}$ has a strictly positive kernel for $N$ large enough. Then the result follows from the Doob-Krein-Rutman Theorem [3].

Let $u \in L_{\tilde{\mu}}^{1}$ be nonnegative and suppose $M \subset \Sigma$ is a set of positive $\tilde{\mu}$-measure such that $\left.u\right|_{M I}>0$. Our aim is to show that $\widetilde{H}^{N} u>0 \tilde{\mu}$-everywhere on $\Sigma$. For that we estimate

$$
\begin{aligned}
\widetilde{H} u & =K \sum_{i=0}^{\infty}((1-\epsilon) K)^{i} \epsilon u \\
& \geq K \epsilon u+K[(1-\epsilon) K]^{N} \epsilon u .
\end{aligned}
$$

Before the general case we distinguish two special cases: $\epsilon \leq \epsilon_{1}<1$ on $\Sigma$ (globally reflecting surfaces) and $0 \leq \epsilon_{0} \leq \epsilon$ on $\Sigma$ (globally absorbing surfaces). In the reflecting case we observe that $K[(1-\epsilon) K]^{N} \epsilon u>0$ on $\Sigma$ for sufficiently large $N$ (iterated reflection). Similarly, if $\epsilon \geq \epsilon_{0}$ we have that $(K \epsilon)^{N} u>0$ for some $N$ (iterated re-emission).

For the general case we have to combine the above cases. Suppose $\widetilde{H}$ is not strictly positive everywhere. This happens only if all possible reflection paths from $M$ to some subset of $\Sigma$ go via the fully absorbing part of $\Sigma$. On the absorbing part we can proceed with the re-emission argument however. Hence we can deduce that $\widetilde{H}^{N} u>0$ on $\Sigma$.

REMARK 3.1. If $\Sigma$ is not an enclosure (i.e., $\mathbb{R}^{n} \backslash \Sigma$ is connected) the analysis can be carried out almost as above. The main difference is that 1 is no longer an eigenvalue of $K$ or $H$, since part of the radiation can escape the system. In fact, this makes the analysis easier as the operators $K$ and $H$ become contractive; see [21]. Moreover, if there is a radiation arriving from outside of the system, additional data terms appear in (3.2) and (3.3).

3.2. Semitransparent materials. Let $\Omega \subset \mathbb{R}^{3}$ be a bounded domain occupied by conductive and semitransparent material and, to simplify presentation, assume additionally that $\Omega$ is convex. In this case, the heat equation is augmented with a volumetric heat sink (or source) $h_{\mathrm{r}}$ due to radiation,

$$
-\nabla \cdot(k \nabla T)=f-h_{\mathrm{r}} \quad \text { in } \Omega .
$$

On the boundary we prescribe

$$
-k \frac{\partial T}{\partial n}=\zeta\left(T-T_{0}\right) \quad \text { on } \partial \Omega
$$

where $T_{0}$ is the exterior temperature. Note that if $\zeta \equiv 0$, then $\mathcal{N}(A)$ is nontrivial and the radiative part has to be analysed carefully in order to satisfy condition (H6).

We derive next the radiative heat source $h_{\mathrm{r}}$. To stress the structural similarity between the semitransparent model and the surface radiation model we redefine some notation used in the previous subsection. $h_{\mathrm{r}}$ is subject to losses by emission, which due to the Stefan-Boltzmann law is proportional to $4 \sigma T^{4}$, and gains by absorption, which is proportional to photon density. The density of photons at $x \in \Omega$ with direction $\omega \in S$ is described by the radiation intensity $u(x, \omega), S$ denoting the surface of the unit sphere. Hence,

$$
h_{\mathrm{r}}(x)=\int_{S} \kappa(x) \frac{\sigma}{\pi} T^{4}(x)-\kappa(x) u(x, \omega) d \omega,
$$


where we have used Kirchoff's law stating that the emission coefficient $\kappa \geq 0$ is equal to the absorption coefficient. The intensity $u$ is governed by the following transport equation:

$$
\begin{aligned}
\omega \cdot \nabla_{x} u+\beta u & =\kappa \frac{\sigma}{\pi} T^{4}+\mathcal{S} u \quad \text { in } \Omega \times S, \\
\left.u\right|_{\Gamma_{-}} & =\mathcal{R}\left(\left.u\right|_{\Gamma_{+}}\right)+q^{\infty},
\end{aligned}
$$

where $q^{\infty}=q^{\infty}(x, \omega)$ accounts for inflow due to external radiation, $\mathcal{S}$ and $\mathcal{R}$ are linear scattering and reflection operators and $\Gamma_{+}, \Gamma_{-}$denote the outflow and inflow boundaries

$$
\begin{aligned}
& \Gamma_{+}=\{(x, \omega) \in \partial \Omega \times S: \omega \cdot n(x)>0\}, \\
& \Gamma_{-}=\{(x, \omega) \in \partial \Omega \times S: \omega \cdot n(x)<0\} .
\end{aligned}
$$

The physical interpretation of the above transport equation is the following: $\beta \geq 0$ is the attenuation coefficient describing the ability of the material to absorb and scatter radiation. On the other hand, intensity is increased by emission (the term $\kappa \sigma T^{4} / \pi$ ) and redirection of radiation due to scattering (the term $\mathcal{S} u$ ). On the boundary, radiation is partly transmitted through and partly reflected back. Here we shall restrict ourselves to the specular reflection operator

$$
(\mathcal{R} u)(x, \omega)=\alpha(x, \omega) u(x, \omega-2(\omega \cdot n) n) \quad(x, \omega) \in \Gamma_{-},
$$

which is both widely used in practice and also mathematically challenging. Another typical and somewhat simpler example is given by the diffusive reflection operator

$$
\left(\mathcal{R}_{d} u\right)(x)=\frac{\alpha(x)}{\pi} \int_{S} u(x, \omega)(\omega \cdot n(x))^{+} d \omega \quad(x, \omega) \in \Gamma_{-},
$$

which we studied in [11]. Also, a linear combination of specular and diffusive reflection is sometimes used in applications. Note that the inflow term $q^{\infty}$ depends on the type of boundary reflections. For example, assuming specular reflections and that the exterior of $\Omega$ is at uniform temperature $T_{0}$ with emission cocfficient $\kappa_{0}$, we have an inflow $q^{\infty}(x, \omega)=$ $(1-\alpha(x, \omega)) \kappa_{0} \sigma T_{0}^{4} / \pi$.

We define the scattering operator as

$$
(\mathcal{S} u)(x, \omega)=\int_{S} \varrho\left(x, \omega^{\prime}, \omega\right) u\left(x, \omega^{\prime}\right) d \omega^{\prime},
$$

where the scattering kernel $\varrho \geq 0$ describing changes from the flow direction $\omega^{\prime}$ to $\omega$ is assumed given. From data of the model we assume that

(i) $0 \leq \alpha \leq \alpha_{1}<1$,

(ii) $\kappa \geq \kappa_{0}>0$ and $\beta$ are bounded,

(iii) $\varrho\left(x, \omega^{\prime}, \omega\right)=\varrho\left(x, \omega, \omega^{\prime}\right)$ for all $\omega, \omega^{\prime} \in S$,

(iv) $q^{\infty} \in L^{6 / 5}\left(\Gamma_{-}\right)$with respect to the measure $(\omega \cdot n)^{-} d \omega d x$ on $\Gamma_{-}$.

Recall also that since no photons are lost, $\kappa, \varrho$ and $\beta$ are related by

$$
\kappa(x)+\int_{S} \varrho\left(x, \omega^{\prime}, \omega\right) d \omega^{\prime}=\beta(x, \omega) .
$$

We must admit that the assumption (i) excludes some physical cases where total reflection occurs near grazing angles (i.e., $\alpha(\omega)=1$ if $|\omega \cdot n|<\delta$ ). To our knowledge, the analysis 
of the radiative transfer equation (3.13)-(3.14) for general specular reflection coefficients is an open question, even in the purely radiative case.

The next step is to reformulate the problem (3.10)-(3.14) to reveal the desired structure. The trick is to define the relative emission coefficient

$$
\epsilon(x, \omega):= \begin{cases}\kappa(x) / \beta(x, \omega) & \text { in } \Omega \times S, \\ 0 & \text { on } \Gamma_{-},\end{cases}
$$

the combined scattering and reflection operator

$$
(R u)(x, \omega):= \begin{cases}\beta^{-1} \mathcal{S} u & \text { if }(x, \omega) \in \Omega \times S, \\ \mathcal{R} u & \text { if }(x, \omega) \in \Gamma_{-},\end{cases}
$$

and a data term

$$
q(x, \omega)= \begin{cases}0 & \text { if }(x, \omega) \in \Omega \times S \\ q^{\infty} & \text { if }(x, \omega) \in \Gamma_{-} .\end{cases}
$$

Now, defining $\rho$ as

$$
\rho=\epsilon \frac{\sigma}{\pi} T^{4}+R u+q \quad \text { in } \Omega \times S \cup \Gamma_{-},
$$

we have

$$
\begin{aligned}
\omega \cdot \nabla_{x} u+\beta u & =\beta \rho \quad \text { in } \Omega \times S, \\
\left.u\right|_{\Gamma_{-}} & =\rho,
\end{aligned}
$$

which can be integrated to get

$$
\begin{aligned}
u(x, \omega)= & \exp \left(-\int_{0}^{d(x, \omega)} \beta(x-s \omega, \omega) d s\right) \rho(x-d(x, \omega) \omega, \omega) \\
& +\int_{0}^{d(x, \omega)} \exp \left(-\int_{0}^{t} \beta(x-s \omega, \omega) d s\right) \beta(x-t \omega, \omega) \rho(x-t \omega, \omega) d t \\
=: & (K \rho)(x, \omega),
\end{aligned}
$$

where $d(x, \omega)$ denotes the distance from $x$ to the inflow boundary in the direction $-\omega$. To summarize,

$$
(I-R K) \rho=\epsilon \frac{\sigma}{\pi} T^{4}+q \quad \text { in } \Omega \times S \cup \Gamma_{-} .
$$

This problem will be analysed with respect to the measures $\nu_{+}$and $\nu_{-}$:

$$
\nu_{ \pm}(B)=\int_{\Lambda \times S \cap B} \beta d \omega d x+\int_{\Gamma_{ \pm} \cap B}(\omega \cdot n)^{ \pm} d \omega d s
$$

for a Borel set $B \subset \mathbb{R}^{3} \times S$.

When analysing $K$ it is often useful to express the solid angle integration in terms of Cartesian coordinates: For $x \in \Omega$ we substitute $y=x-t \omega$ and $\omega=(x-y) /|x-y|$ so 
that $d t d \omega=|x-y|^{-2} d x$ and further, for $s=x-d \omega \in \partial \Omega$ the solid angle transforms into $d \omega=n(s) \cdot(x-s)|x-s|^{-3} d s$. Therefore,

$$
\begin{aligned}
\int_{S}(K u)(x, \omega) d \omega= & \int_{\Omega} \frac{\tau(x, y)}{|x-y|^{2}} \beta(y) u\left(y, \frac{x-y}{|x-y|}\right) d y \\
& +\int_{\partial \Omega} \frac{\tau(x, s) n(s) \cdot(x-s)}{|x-s|^{3}} u\left(s, \frac{x-s}{|x-s|}\right) d s
\end{aligned}
$$

where

$$
\tau(x, y)=\exp \left(-\int_{0}^{|x-y|} \beta\left(x-t \frac{x-y}{|x-y|}\right) d t\right),
$$

and similarly, for $z \in \partial \Omega$,

$$
\begin{aligned}
\int_{S}(K u)(z, \omega)(\omega \cdot n(z))^{+} d \omega= & \int_{\Omega \Omega} \frac{\tau(z, y) n(z) \cdot(y-z)}{|z-y|^{3}} \beta(y) u\left(y, \frac{z-y}{|z-y|}\right) d y \\
& +\int_{\partial \Omega} \frac{\tau(z, s) n(s) \cdot(z-s) n(z) \cdot(s-z)}{|z-s|^{4}} u\left(s, \frac{z-s}{|z-s|}\right) d s .
\end{aligned}
$$

Note that for nonconvex $\Omega$, the visibility factor defined in (3.5) has to be added in the definition of $\tau(x, y)$.

Lemma 4 . Let $1 \leq p \leq \infty$. The operator $K$ maps $L_{\nu^{-}}^{p}$ to $L_{\nu^{+}}^{p}$ with $K 1=1$ and $\|K\|=1$. The adjoint operator $K^{*}: L_{\nu_{+}}^{q} \mapsto L_{\nu_{-}}^{q}(q$ denoting the conjugate of $p)$ satisfies also $K^{*} 1=1$ and reads as

$$
\begin{aligned}
\left(K^{*} v\right)(x, \omega)= & \exp \left(-\int_{0}^{d^{*}(x, \omega)} \beta(x+s \omega, \omega) d s\right) v\left(x+d^{*}(x, \omega) \omega, \omega\right) \\
& +\int_{0}^{d^{*}(x, \omega)} \exp \left(-\int_{0}^{t} \beta(x+s \omega, \omega) d s\right) \beta(x+t \omega, \omega) v(x+t \omega, \omega) d t
\end{aligned}
$$

$d^{*}(x, \omega)$ denoting the distance of $x$ from the boundary in the direction $\omega$.

Proof. The statement $K 1=1=K^{*} 1$ follows by direct integration.

To derive the adjoint operator $K^{*}$, let $p$ and $q$ be conjugate exponents and take $u \in L_{\nu_{-}}^{p}$ and $v \in L_{\nu_{+}}^{q}$. Then, writing the solid angle integrals in $\int v K u d \nu_{+}$in Cartesian form as above, changing the order of integration of the Cartesian integrals and transforming back to solid angle integration yields, after some tedious manipulations, $\int u K^{*} v d \nu_{-}$.

To avoid lengthy notation, we shall estimate the norm of $K$ when $\beta$ is constant and $1<p<\infty$. The extension to the general case is straightforward. Using the standard mollifier $\eta_{\varepsilon}(t)$ in $\mathbb{R}$ (i.e., $\eta_{\varepsilon}: \mathbb{R} \rightarrow \mathbb{R}$ is a $C^{\infty}$-function such that $\int \eta_{\varepsilon}(s-t) f(t) d t \rightarrow f(s)$ as $\varepsilon \rightarrow 0$ ), we may write

$$
e^{-\beta d} u(x-d \omega)=\int_{0}^{d} \eta_{\varepsilon}(d-t) e^{-\beta d} u(x-t \omega) d t+f_{\varepsilon}
$$


where $f_{\varepsilon} \rightarrow 0$ as $\varepsilon \rightarrow 0$. Hence,

$$
\begin{aligned}
|(K u)(x, \omega)| \leq & \int_{0}^{d}\left(\beta+\eta_{\varepsilon}(d-t)\right) e^{-\beta d}|u(x-t \omega)| d t+\left|f_{\varepsilon}\right| \\
\leq & \left(\int_{0}^{d}\left(\beta+\eta_{\varepsilon}(d-t)\right) e^{-\beta d} d t\right)^{1-\frac{1}{p}} \\
& \times\left(\int_{0}^{d}\left(\beta+\eta_{\varepsilon}(d-t)\right)|u(x-t \omega)|^{p} d t\right)^{\frac{1}{p}}+\left|f_{\varepsilon}\right| \\
& \rightarrow(K 1)^{1-\frac{1}{p}}\left(K|u|^{p}\right)^{\frac{1}{p}} \quad \text { as } \varepsilon \rightarrow 0 .
\end{aligned}
$$

Thus,

$$
\int|K u|^{p} d \nu_{+} \leq \int K|u|^{p} d \nu_{+}=\int|u|^{p} K^{*} 1 d \nu_{-}=\int|u|^{p} d \nu_{-} .
$$

Lemma 5. Let $1 \leq p \leq \infty$. The operator $I-R K$ from $L_{\nu_{-}}^{p}$ to itself is invertible and this inverse is positive.

Proof. From assumptions (i) and (ii) on the coefficients $\kappa, \beta, \alpha$, in conjunction with relation $(3.18)$, it follows that

$$
R 1 \leq \max \left\{1-\kappa_{0} /\|\beta\|_{\infty}, \alpha_{1}\right\}<1 .
$$

Since $\varrho$ is symmetric by assumption (iii), also $R^{*} 1<1$, and, hence the argument that was used to analyse the norm of $K$ shows that $\|R\|<1$. Thus, $I-R K$ is invertible and, writing this inverse as a Neumann series, we notice that it is also positive.

Now, we are ready to identify the operators $E, F, G$ and the measure $\mu$. First, resolving $\rho$ from (3.24) and writing $\beta=\beta \cdot \kappa / \beta=\beta \epsilon$ we have

$$
\kappa u=\kappa K \rho=\frac{\sigma}{\pi} \beta \epsilon K(I-R K)^{-1} \epsilon T^{4}+\tilde{q},
$$

with $\tilde{q}=\kappa K(I-R K)^{-1} q$. In the light of (3.12) we thus have

$$
h_{\mathrm{r}}=\frac{\sigma}{\pi} \int_{S} \beta G_{\omega} T^{4} d \omega-\int_{S} \tilde{q} d \omega
$$

where $G_{\omega} T^{4}=\epsilon T^{4}-\epsilon K(I-R K)^{-1} \epsilon T^{4}$. This expression still needs to be scaled in order to satisfy the hypotheses (H3)-(H5). For this reason, we define the operators $G$ and $F$ as

$$
\begin{aligned}
G T^{4} & =\frac{1}{4 \kappa \pi} \int_{S} \beta G_{\omega} T^{4} d \omega \\
F T^{4} & =\frac{1}{4 \kappa \pi} \int_{S} \beta \epsilon K(I-R K)^{-1} \epsilon T^{4} d \omega .
\end{aligned}
$$

In this formulation $E$ is an identity operator since $\int_{S} \beta \epsilon d \omega /(4 \kappa \pi)=1$. Finally, defining the measure $d \mu=4 \sigma \kappa d x$ we have

$$
\int_{\Omega} h_{\mathrm{r}}(T) \varphi d x=\int_{\Omega} G T^{4} \varphi d \mu-\int_{\Omega}\left(\int_{S} \tilde{q} d \omega\right) \varphi d x .
$$


Since $L_{\mu}^{5} \subset H^{1}(\Omega)$ we now have $V=H^{1}(\Omega)$. Note also that the data term is well defined since $q^{\infty} \in L^{6 / 5}\left(\Gamma_{-}\right)$implies $\int_{S} \tilde{q} d \omega \in L^{6 / 5}(\Omega)$ and $\varphi \in L^{6}(\Omega) \subset H^{1}(\Omega)$.

The remaining hypotheses $(\mathrm{H} 3)-(\mathrm{H} 7)$ are verified in the following lemma.

Lemma 6. Both $G 1$ and $G^{*} 1$ are strictly positive and $\mathcal{N}(G)=\{0\}$. Furthermore, the operator $F: L_{\mu}^{p} \mapsto L_{\mu}^{p}$ is positive and compact for $1<p<\infty$.

Proof. Writing the inverse term in $G_{\omega}$ as a Neumann series, we notice that

$$
G_{\omega}=\epsilon-\epsilon(I-K R)^{-1} K \epsilon=\epsilon(I-K R)^{-1}(I-K(R+\epsilon)) .
$$

Furthermore, using the relation (3.18) and the assumption $\alpha \leq \alpha_{1}<1$, we see that $K(R 1+\epsilon)<1$, and, hence

$$
G 1 \geq \frac{\kappa_{0}}{4 \pi \kappa} \int_{S} 1-K(R 1+\epsilon) d \omega>0
$$

since $\epsilon(I-K R)^{-1}$ is a positive operator. Similarly we see that $G^{*} 1>0$. Now, since both $H 1$ and $H^{*} 1$ are strictly less than one and $H, H^{*}$ are positive, we may argue as in Lemma 8 to get $\|H\|<1$, which implies that the null space of $G$ is trivial.

To show that $F$ is compact, we analyse the operator $\widetilde{K}: L_{\nu_{-}}^{p} \rightarrow L^{p}(\Omega)$,

$$
(\widetilde{K} v)(x):=\int_{S} K v d w
$$

This operator has integrable kernel, and hence its compactness can be established as for the weakly singular operators [8]: we define an operator $\widetilde{K}_{\varepsilon}$ by truncating the kernel of $\widetilde{K}$ in the $\varepsilon$-neighborhood of the singularity. Then, $\widetilde{K}_{\varepsilon}$ is compact since it has a bounded kernel. Furthermore, $\left\|\widetilde{K}_{\varepsilon}-\widetilde{K}\right\|$ tends to zero as $\varepsilon \rightarrow 0$, since the kernel of $\widetilde{K}$ is integrable.

REMARK 3.2. With minor modifications, the above analysis can be carried out for a class of reflection operators. Then, we only need to assume that the reflection operator $\mathcal{R}$ is a positive and contractive mapping from $L^{p}\left(\Gamma_{+}\right)$to $L^{p}\left(\Gamma_{-}\right)$(defining these spaces with measures $\left.(\omega \cdot n)^{ \pm} d \omega d x\right)$.

REMARK 3.3. In derivation of the above model we implicitly assumed that the radiation is unpolarized. However, if polarization effects are important, they can be taken into account by replacing the directional intensity $u(x, \omega)$ by a more general quantity $u(x, \omega, \phi)$ where $\phi \in[0, \pi)$ is the polarization angle. Naturally, also the relative emissivity $\epsilon$ has to be defined for all polarization angles as well as the scattering/reflection operator $R$ which now has the kernel defined in $\Omega \times S \times S \cup \Gamma_{-} \times[0, \pi) \times[0, \pi)$. Definition of the relevant function spaces and measures is straightforward.

3.3. Models with several components. We present next a convenient way to characterise condition (H6) in the presence of several conducting and radiating components.

We form a graph whose nodes are the conducting components $\Omega_{i}$ of the system and link two nodes if they are in contact by radiation. Further, we say that the conductive and radiative system is in contact with the environment if one of the following holds:

(i) $\Gamma$ has a positive surface measure,

(ii) temperature is prescribed on part of $\partial \Omega$ (see Remark 2.1),

(iii) there is a set $Z \subset \Lambda \cup \Sigma$ such that $\left.u\right|_{Z} \equiv 0$ for all $u \in \mathcal{N}(G)$. 

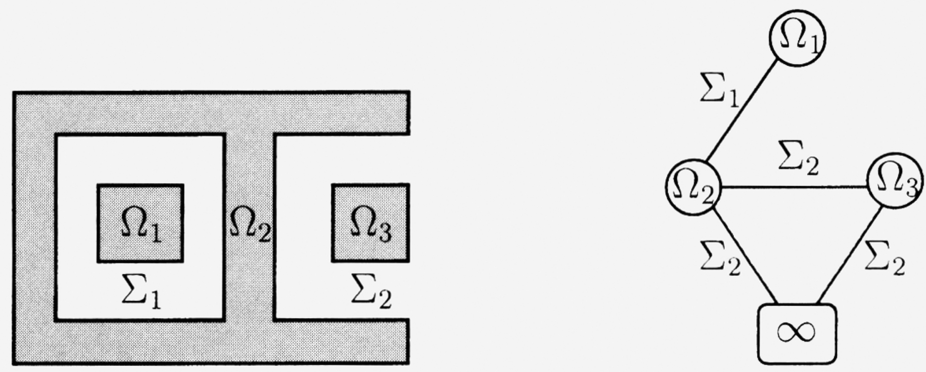

FIG. 2. System with three conducting and two radiating components

We note that in practice (iii) means that part of the radiation is escaping the system. Now, it is easily seen that the condition (H6) holds if the following two conditions are fulfilled: (i) the graph describing the system is connected and (ii) the system is in contact with the environment.

Figure 2 shows a situation that is sufficiently rich to illustrate the above ideas. Here $\Omega=\Omega_{1} \cup \Omega_{2} \cup \Omega_{3}$ is opaque, $\Sigma=\Sigma_{1} \cup \Sigma_{2}$ is diffuse-grey, $\Lambda=\varnothing$, and " $\infty$ " denotes the environment. First, radiative transfer has to be modelled separately on $\Sigma_{1}$ and $\Sigma_{2}$ giving the operators $H_{1}$ and $H_{2}$. Then, by setting $(H u)(x)=\left(H_{i} u\right)(x)$ for $x \in \Sigma_{i}$, the properties (H2)-(H5) of $H$ follow from the corresponding properties of the operators $H_{i}$. Note that the surface $\Sigma_{2}$ is open and hence 1 is not an eigenvalue of $H_{2}$; see [21]. Consequently, the condition ( $\mathrm{H} 6)$ is now fulfilled no matter which boundary condition is prescribed on the outer boundary of $\Omega_{2}$.

4. Existence of solutions. In this section we prove that the operator $Q: V \rightarrow V^{*}$ is pseudomonotone and coercive. These properties imply the following existence result; see Zeidler [23].

Theorem 2. Under the hypotheses of Sec. 2, there exists a solution to (2.2).

Lemma 7. The operator $Q: V \rightarrow V^{*}$ is pseudomonotone, that is,

$$
u_{i} \rightarrow u \text { weakly in } V \text { and } \varlimsup_{i \rightarrow \infty}\left\langle Q u_{i}, u_{i}-u\right\rangle \leq 0
$$

imply

$$
\langle Q u, u-v\rangle \leq \underline{\lim }_{i \rightarrow \infty}\left\langle Q u_{i}, u_{i}-v\right\rangle \quad \forall v \in V .
$$

Proof. According to (H5) we can write $G=E-F$ where $F$ is compact in $L_{\mu}^{5 / 4}$. Due to monotonicity of the operator

$$
\langle\widetilde{A} u, v\rangle:=\langle A u, v\rangle+\left\langle E|u|^{3} u, v\right\rangle_{\mu} \quad \forall v \in V,
$$

it suffices to prove that the mapping $u \mapsto F|u|^{3} u$ is pseudomonotone in $V$.

Let $u_{i} \rightarrow u$ weakly in $V$. Then $u_{i} \rightarrow u$ weakly in $L_{\mu}^{5}$ and $u_{i} \rightarrow u$ weakly in $H^{1}(\Omega)$. Thus $u_{i} \rightarrow u$ strongly in $L_{\mu}^{2}$ since the embeddings $H^{1}(\Omega) \subset L^{2}(\Lambda)$ and $H^{1}(\Omega) \subset L^{2}(\Sigma)$ are compact; see [14]. Consequently, $u_{i} \rightarrow u \mu$-a.e. in $\Lambda \cup \Sigma$ and hence also $\left|u_{i}\right|^{3} u_{i} \rightarrow|u|^{3} u$ 
$\mu$-a.e. Therefore, $\left|u_{i}\right|^{3} u_{i} \rightarrow|u|^{3} u$ weakly in $L_{\mu}^{5 / 4}$ since the sequence $\left\{\left|u_{i}\right|^{3} u_{i}\right\}$ is bounded in $L_{\mu}^{5 / 4}$. Finally, the compactness of $F$ implies

$$
\begin{aligned}
& \left\langle F|u|^{3} u, u-v\right\rangle_{\mu}-\left\langle F\left|u_{i}\right|^{3} u_{i}, u_{i}-v\right\rangle_{\mu} \\
& \quad=\left\langle F\left(|u|^{3} u-\left|u_{i}\right|^{3} u_{i}\right), u_{i}-v\right\rangle_{\mu}-\left\langle F|u|^{3} u, u-u_{i}\right\rangle_{\mu} \rightarrow 0
\end{aligned}
$$

for all $v \in V$.

The coercivity in the space $L_{\mu}^{5}$ is characterized by the following two lemmas.

LEMma 8. $\|H\|_{L_{\mu}^{p}} \leq 1$ for $1 \leq p \leq \infty$ and $\left\langle G|u|^{3} u, u\right\rangle_{\mu} \geq 0$ for all $u \in L_{\mu}^{5}$.

Proof. Let $u \in L_{\mu}^{1}$ be nomegative. Then

$$
\int H u d \mu=\int u H^{*} 1 d \mu \leq \int u d \mu .
$$

Since $H$ is positive, this implies $\|H\|_{L_{\mu}^{1}} \leq 1$. On the other hand, $H\left(1-v /\|v\|_{L_{\mu}^{\infty}}\right) \geq 0$ and thus $\|H\|_{L_{\mu}^{\infty}} \leq\|H 1\|_{L_{\mu}^{\infty}} \leq 1$. Now, from the Riesz interpolation theorem, it follows that $\|H\|_{L_{\mu}^{p}} \leq 1,1 \leq p \leq \infty$; see $[1]$ for example.

The second result follows from the Hölder inequality

$$
\left\langle G|u|^{3} u, u\right\rangle_{\mu} \geq\|u\|_{L_{\mu}^{5}}^{5}-\left\|H|u|^{3} u\right\|_{L_{\mu}^{5 / 4}}\|u\|_{L_{\mu}^{5}} \geq(1-\|H\|)\|u\|_{L_{\mu}^{5}}^{5} \geq 0 .
$$

Lemma 9. If $u \in L_{\mu}^{5}$ and $u \notin \mathcal{N}(G)$, then $\left\langle G|u|^{3} u, u\right\rangle_{\mu}>0$.

Proof. Using positivity of $H$, we notice that

$$
\left\langle G|u|^{3} u, u\right\rangle_{\mu} \geq\left\langle G u_{+}^{4}, u_{+}\right\rangle_{\mu}+\left\langle G u_{-}^{4}, u_{-}\right\rangle_{\mu} .
$$

Hence, with no loss of generality, we may assume $u \geq 0$ and $\|u\|_{L_{\mu}^{5}}=1$. We utilize the Riesz interpolation technique for positive integral operators [7] to show that

$$
\left\langle H u^{4}, u\right\rangle_{\mu}<\left\langle u^{4}, u\right\rangle_{\mu}=\|u\|_{L_{\mu}^{5}}^{5} \quad \text { if } u \notin \mathcal{N}(G) .
$$

Since $F$ is compact it can be presented as an integral operator [22]. Further, using the standard mollifier of $(1-e) u$ we may write $H u$ as

$$
H u=(1-c) u+F u=\lim _{\varepsilon \rightarrow 0} \int h_{\varepsilon}(x, y) u(y) d \mu(y)
$$

with $h_{\varepsilon} \geq 0$ for all $\varepsilon$.

Next let $p_{1}=6 / 5, p=5 / 4, p_{2}=2$ and denote by $q_{1}, q, q_{2}$ the corresponding conjugate exponents. Further, choose $\alpha=9 / 10$ so that $1 / p=\alpha / p_{1}+(1-\alpha) / p_{2}$. Then, for $u, v \geq 0$, the Hölder inequality gives

$$
\begin{aligned}
\int u\left(\int h_{\varepsilon} v d \mu\right) d \mu & =\int u\left(\int h_{\varepsilon}^{\alpha+(1-\alpha)} v^{p\left(\frac{\alpha}{p_{1}}+\frac{1-\alpha}{p_{2}}\right)} d \mu\right) d \mu \\
& \leq \int u\left(\int h_{\varepsilon} v^{\frac{p}{p_{1}}} d \mu\right)^{\alpha}\left(\int h_{\varepsilon} v^{\frac{p}{p_{2}}} d \mu\right)^{1-\alpha} d \mu \\
& \leq\left(\int u^{\frac{q}{q_{1}}} \int h_{\varepsilon} v^{\frac{p}{p_{1}}} d \mu d \mu\right)^{\alpha}\left(\int u^{\frac{q}{q_{2}}} \int h_{\varepsilon} v^{\frac{p}{p_{2}}} d \mu d \mu\right)^{1-\alpha} .
\end{aligned}
$$

Consequently, taking the limit $\varepsilon \rightarrow 0$,

$$
\langle u, H v\rangle_{\mu} \leq\left\langle u^{\frac{q}{q_{1}}}, H v^{\frac{p}{p_{1}}}\right\rangle_{\mu}^{\alpha}\left\langle u^{\frac{q}{q_{2}}}, H v^{\frac{p}{p_{2}}}\right\rangle_{\mu}^{1-\alpha} .
$$


Now, for $v=u^{4}$ we obtain

$$
\left\langle u^{\frac{q}{q_{1}}}, H v^{\frac{p}{p_{1}}}\right\rangle_{\mu} \leq\left\|u^{\frac{q}{q_{1}}}\right\|_{L_{\mu}^{q_{1}}}^{\frac{q}{q_{1}}}\left\|v^{\frac{p}{p_{1}}}\right\|_{L_{\mu}^{p_{1}}}^{\frac{p}{p_{1}}} \leq\|u\|_{L_{\mu}^{q}}^{\frac{q}{q_{1}}}\left\|u^{4}\right\|_{L_{\mu}^{p}}^{\frac{p}{p_{1}}}=\|u\|_{L_{\mu}^{5}}^{5}=1,
$$

and $\left\langle u^{\frac{q}{q_{2}}}, H v^{\frac{p}{p_{2}}}\right\rangle_{\mu}=\left\langle u^{\frac{5}{2}}, H u^{\frac{5}{2}}\right\rangle_{\mu}$ so that

$$
\left\langle u, H u^{4}\right\rangle_{\mu} \leq\left\langle u^{\frac{5}{2}}, H u^{\frac{5}{2}}\right\rangle_{\mu} .
$$

Suppose next $\left\langle u^{\frac{5}{2}}, H u^{\frac{5}{2}}\right\rangle_{\mu}=\|u\|_{L_{\mu}^{5}}^{5}$. Then, denoting $v=u^{\frac{5}{2}}$, we have

$$
0=\langle v, v-H v\rangle_{\mu} \geq\|v\|_{L_{\mu}^{2}}^{2}-\|H v\|_{L_{\mu}^{2}}\|v\|_{L_{\mu}^{2}}
$$

and so $\|H v\|_{L_{\mu}^{2}}=\|v\|_{L_{\mu}^{2}}$. Therefore, since

$$
\left\langle v,\left(I-H^{*} H\right) v\right\rangle_{\mu}=\langle v, v\rangle_{\mu}-\langle H v, H v\rangle_{\mu}=0
$$

we have

$$
\|G v\|_{L_{\mu}^{2}}^{2}=\left\langle v, G^{*} G v\right\rangle_{\mu}=\left\langle v,(I-H) v+\left(I-H^{*}\right) v-\left(I-H^{*} H\right) v\right\rangle_{\mu}=0,
$$

which implies $u^{\frac{5}{2}}=v \in \mathcal{N}(G)$ and hence also $u \in \mathcal{N}(G)$ according to (H7). Therefore, if $u \notin \mathcal{N}(G)$, then inequalities (4.3) and (4.2) are strict.

TheOREM 3. There exists $C>0$ such that

$$
\langle Q u, u\rangle \geq C \min \left\{\|u\|_{V}^{2},\|u\|_{V}^{q}\right\} \quad \forall u \in V,
$$

where $q=\max \{p+1,5\}$.

Proof. Suppose first that (4.4) is not true if $\|u\|_{V} \geq 1$. Then, for each integer $i$ there is $\tilde{u}_{i} \in V$ such that $\left\|\tilde{u}_{i}\right\|_{V} \geq 1$ and

$$
\left\|\tilde{u}_{i}\right\|_{V}^{2}>i\left\langle Q \tilde{u}_{i}, \tilde{u}_{i}\right\rangle .
$$

The sequence $u_{i}=\tilde{u}_{i} /\left\|\tilde{u}_{i}\right\|_{V}$ satisfies $\left\|u_{i}\right\|_{V}=1$ and

$$
\begin{aligned}
\left\|u_{i}\right\|_{V}^{2} & >i C \\
\geq & \left\{\int_{\Omega}\left|\nabla u_{i}\right|^{2} d x+\left\|\tilde{u}_{i}\right\|_{V}^{p-1} \int_{\Gamma}\left|u_{i}\right|^{p+1} d s+\left\|\tilde{u}_{i}\right\|_{V}^{3}\left\langle G\left|u_{i}\right|^{3} u_{i}, u_{i}\right\rangle_{\mu}\right\} \\
\geq & \left\{\int_{\Omega}\left|\nabla u_{i}\right|^{2} d x+\int_{\Gamma}\left|u_{i}\right|^{p+1} d s+\left\langle G\left|u_{i}\right|^{3} u_{i}, u_{i}\right\rangle_{\mu}\right\}
\end{aligned}
$$

according to (2) and Lemma 8. Since $\left\{u_{i}\right\}$ is bounded in $V$, there is a subsequence (still denoted by $u_{i}$ ) and $u \in V$ such that $u_{i}$ converges weakly to $u$ in the spaces $L_{\mu}^{5}, H^{1}(\Omega)$, and $L^{p+1}(\Gamma)$. Furthermore, $u_{i} \rightarrow u$ strongly in $L^{2}(\Omega)$, since the embedding $H^{1}(\Omega) \subset L^{2}(\Omega)$ is compact. Since $\left\|u_{i}\right\|_{V}=1$, from (4.5) it follows that $\left\|\nabla u_{i}\right\|_{L^{2}(\Omega)} \rightarrow 0$. Consequently, $\nabla u \equiv 0$ and therefore $u$ is a constant individually in each connected component of $\Omega$. Hence we have also $u_{i} \rightarrow u$ strongly in $H^{1}(\Omega)$. Now (4.5) implies that

$$
\|u\|_{L^{p+1}(\Gamma)} \leq \underline{\lim }_{i \rightarrow \infty}\left\|u_{i}\right\|_{L^{p+1}(\Gamma)}=\lim _{i \rightarrow \infty}\left\|u_{i}\right\|_{L^{p+1}(\Gamma)}=0 .
$$

Thus $\left.u\right|_{\Gamma}=0$ and from the Radon-Riesz Theorem we get $u_{i} \rightarrow u$ strongly in $L^{p+1}(\Gamma)$. Applying once more (4.5), we discover that

$$
\left\langle G\left|u_{i}\right|^{3} u_{i}, u_{i}\right\rangle_{\mu} \rightarrow 0 .
$$


Since $u_{i} \rightarrow u$ weakly in $L_{\mu}^{5}$, we have $\left|u_{i}\right|^{3} u_{i} \rightarrow|u|^{3} u$ weakly in $L_{\mu}^{5 / 4}$ and thus

$$
\begin{aligned}
& \left\langle F\left|u_{i}\right|^{3} u_{i}, u_{i}\right\rangle_{\mu}-\left\langle F|u|^{3} u, u\right\rangle_{\mu} \\
& \quad=\left\langle F\left|u_{i}\right|^{3} u_{i}-F|u|^{3} u, u_{i}\right\rangle_{\mu}-\left\langle F|u|^{3} u, u_{i}-u\right\rangle_{\mu} \\
& \quad \leq\left\|F\left|u_{i}\right|^{3} u_{i}-F|u|^{3} u\right\|_{L_{\mu}^{5 / 4}}\left\|u_{i}\right\|_{L_{\mu}^{5}}+\|u\|_{L_{\mu}^{5}}^{4}\left\|F^{*}\left(u_{i}-u\right)\right\|_{L_{\mu}^{5}} \rightarrow 0
\end{aligned}
$$

since $F$ is compact (hence also $F^{*}$ is compact). $u_{i} \rightarrow u$ weakly in $L_{\mu}^{5}$ implies also $\left\|e^{1 / 5} u\right\|_{L_{\mu}^{5}} \leq \underline{\lim }_{i \rightarrow \infty}\left\|e^{1 / 5} u_{i}\right\|_{L_{\mu}^{5}}$ and therefore, from (4.6) and (4.7),

$$
\begin{aligned}
0=\lim _{i \rightarrow \infty}\left\langle G\left|u_{i}\right|^{3} u_{i}, u_{i}\right\rangle_{\mu} & \geq \underline{\lim }_{i \rightarrow \infty}\left\langle E\left|u_{i}\right|^{3} u_{i}, u_{i}\right\rangle_{\mu}-\underline{\lim }_{i \rightarrow \infty}\left\langle F\left|u_{i}\right|^{3} u_{i}, u_{i}\right\rangle_{\mu} \\
& \geq\left\langle E|u|^{3} u, u\right\rangle_{\mu}-\left\langle F|u|^{3} u, u\right\rangle_{\mu}=\left\langle G|u|^{3} u, u\right\rangle_{\mu} .
\end{aligned}
$$

According to Lemma 9, this implies that $u \in \mathcal{N}(G)$. But since also $u \in \mathcal{N}(A)$, condition (H6) implies $u \equiv 0$. Furthermore,

$$
\left\|e^{1 / 5} u_{i}\right\|_{L_{\mu}^{5}}^{5}=\left\langle G\left|u_{i}\right|^{3} u_{i}, u_{i}\right\rangle_{\mu}-\left\langle F\left|u_{i}\right|^{3} u_{i}, u_{i}\right\rangle_{\mu} \rightarrow 0
$$

according to (4.6) and (4.7). Hence $u_{i} \rightarrow 0$ strongly in $L_{\mu}^{5}$. Since $u_{i} \rightarrow 0$ strongly also in $H^{1}(\Omega)$ and $L^{p+1}(\Gamma)$, we have $u_{i} \rightarrow 0$ strongly in $V$. This is a contradiction since $\left\|u_{i}\right\|_{V}=1$ for every $i$. The proof for $\|u\|_{V}<1$ is similar; we only need to replace the left-hand side of (4.5) with $\|u\|_{V}^{q}$.

REMARK 4.1. The left-hand side of (4.4) has a nonstandard form because different heat transfer mechanisms in (2.2) are comparable to different powers of temperature. For any $\alpha>0$, it is straightforward to modify the proof of Theorem 3 to show that there exists $C>0$ such that

$$
\|u\|_{V}^{\alpha} \leq C\left\{\left(\int_{\Omega} a_{i j} \partial_{i} u \partial_{j} u d x\right)^{\frac{\alpha}{2}}+\left(\int_{\Gamma} \zeta|u|^{p+1} d s\right)^{\frac{\alpha}{p+1}}+\left(\int_{\widetilde{\Omega}} G|u|^{3} u d \mu\right)^{\frac{\alpha}{5}}\right\},
$$

for all $u \in V$.

5. Comparison principle. We prove next that our problem satisfies the so-called comparison principle and has a unique solution. The idea of the proof is borrowed from Křižzek and Liu [10] who analysed a heat conduction problem with a Lipschitz nonlinearity. In contrast to [10], we have to deal now with a nonlocal problem and power-type nonlinearity, which makes the modification of the proof nontrivial. In what follows, we denote the Lebesgue measure in $\mathbb{R}^{n}$ by $\mathcal{L}$.

THEOREM 4. Let $u_{1}$ and $u_{2}$ be solutions of $(2.2)$, corresponding to right-hand sides $f_{1}, f_{2} \in V^{*}$, and assume that

$$
\left\langle f_{1}-f_{2}, v\right\rangle \geq 0 \quad \forall v \geq 0, v \in V .
$$

Then $u_{1} \geq u_{2} \mathcal{L}$-a.e. in $\Omega$, $\gamma$-a.e. on $\Gamma$ and $\mu$-a.e. in $\Lambda \cup \Sigma$. Consequently, the solution of (2.2) is unique. 
Proof. We introduce first some notation and then sketch the main ingredients of the proof. For $\varepsilon>0$ we denote

$$
\begin{aligned}
\Omega_{0} & =\left\{x \in \bar{\Omega}: u_{1}(x)<u_{2}(x)\right\}, \\
\Omega_{\varepsilon} & =\left\{x \in \Omega_{0}: u_{2}(x)-u_{1}(x)>\varepsilon\right\}, \\
v_{\varepsilon} & =\min \left\{\varepsilon,\left(u_{2}-u_{1}\right)^{+}\right\} .
\end{aligned}
$$

We need to prove that $\mu\left(\Omega_{0}\right)+\mathcal{L}\left(\Omega_{0}\right)+\gamma\left(\Omega_{0}\right)=0$. We argue by contradiction and claim first that $\mu\left(\Omega_{0}\right)>0$. The contradiction is shown as follows: First, Theorem 3 and Remark 4.1 imply

$$
\left\|v_{\varepsilon}\right\|_{L_{\mu}^{5}}^{2} \leq C\left\{\int_{\Omega} a_{i j} \partial_{i} v_{\varepsilon} \partial_{j} v_{\varepsilon} d x+\left(\int_{\Gamma} \zeta\left|v_{\varepsilon}\right|^{p+1} d s\right)^{\frac{2}{p+1}}+\left(\int_{\Lambda \cup \Sigma} G v_{\varepsilon}^{4} v_{\varepsilon} d \mu\right)^{\frac{2}{5}}\right\} .
$$

The next step is to estimate

$$
\begin{array}{r}
\int_{\Omega} a_{i j} \partial_{i} v_{\varepsilon} \partial_{j} v_{\varepsilon} d x \leq \varepsilon\left\|v_{\varepsilon}\right\|_{L_{\mu}^{5}} f_{\varepsilon}-g_{\varepsilon}, \\
\left(\int_{\Gamma} \zeta\left|v_{\varepsilon}\right|^{p+1} d s\right)^{\frac{2}{p+1}}+\left(\int_{\tilde{\Omega}} G v_{\varepsilon}^{4} v_{\varepsilon} d \mu\right)^{\frac{2}{5}} \leq \varepsilon\left\|v_{\varepsilon}\right\|_{L_{\mu}^{5}} f_{\varepsilon}+h_{\varepsilon},
\end{array}
$$

where $f_{\varepsilon} \rightarrow 0$ as $\varepsilon \rightarrow 0$ and $h_{\varepsilon}-g_{\varepsilon}$ can be ignored when $\varepsilon$ is small enough. Finally, these estimates give

$$
\mu\left(\Omega_{\varepsilon}\right) \leq \varepsilon^{-1}\left(\int_{\Omega_{\varepsilon}} \varepsilon^{5} d \mu\right)^{\frac{1}{5}} \leq \varepsilon^{-1}\left\|v_{\varepsilon}\right\|_{L_{\mu}^{5}} \leq f_{\varepsilon} \rightarrow 0
$$

which leads to a contradiction. Similarly we can prove that $\mathcal{L}\left(\Omega_{0}\right)=\gamma\left(\Omega_{0}\right)=0$.

1. Derive the estimate (5.1):

$$
\begin{aligned}
\int_{\Omega} a_{i j} \partial_{i} v_{\varepsilon} \partial_{j} v_{\varepsilon} d x= & \int_{\Omega} a_{i j} \partial_{i}\left(u_{2}-u_{1}\right) \partial_{j} v_{\varepsilon} d x \\
= & \left\langle f_{2}-f_{1}, v_{\varepsilon}\right\rangle-\int_{\Gamma} \zeta\left(\left|u_{2}\right|^{p-1} u_{2}-\left|u_{1}\right|^{p-1} u_{1}\right) v_{\varepsilon} d s \\
& +\int_{\Lambda \cup \Sigma} G\left(\left|u_{1}\right|^{3} u_{1}-\left|u_{2}\right|^{3} u_{2}\right) v_{\varepsilon} d \mu
\end{aligned}
$$

The last term in (5.4) can be decomposed as

$$
\begin{aligned}
\int_{\Lambda \cup \Sigma} G\left(\left|u_{1}\right|^{3} u_{1}-\left|u_{2}\right|^{3} u_{2}\right) v_{\varepsilon} d \mu= & \int_{(\Lambda \cup \Sigma) \backslash \Omega_{0}}\left(\left|u_{1}\right|^{3} u_{1}-\left|u_{2}\right|^{3} u_{2}\right) G^{*} v_{\varepsilon} d \mu \\
& +\int_{\Omega_{0} \backslash \Omega_{\varepsilon}}\left(\left|u_{1}\right|^{3} u_{1}-\left|u_{2}\right|^{3} u_{2}\right) G^{*} v_{\varepsilon} d \mu \\
& +\int_{\Omega_{\varepsilon}}\left(\left|u_{1}\right|^{3} u_{1}-\left|u_{2}\right|^{3} u_{2}\right) G^{*} v_{\varepsilon} c^{\prime} \mu
\end{aligned}
$$

The first term in the right-hand side is nonpositive since $\left|u_{1}\right|^{3} u_{1}-\left|u_{2}\right|^{3} u_{2} \geq 0$ and $G^{*} v_{\varepsilon}=0-H^{*} v_{\varepsilon} \leq 0$ in $(\Lambda \cup \Sigma) \backslash \Omega_{0}$. To investigate the second term, we note that

$$
\left|u_{2}\right|^{3} u_{2}-\left|u_{1}\right|^{3} u_{1} \leq\left(u_{2}-u_{1}\right) P\left(\left|u_{2}\right|,\left|u_{1}\right|\right),
$$


where $P(x, y)=x^{3}+x^{2} y+x y^{2}+y^{3}$. Thus

$$
\begin{aligned}
\int_{\Omega_{0} \backslash \Omega_{\varepsilon}}\left(\left|u_{1}\right|^{3} u_{1}-\left|u_{2}\right|^{3} u_{2}\right) G^{*} v_{\varepsilon} d \mu & \leq \int_{\Omega_{0} \backslash \Omega_{\varepsilon}}\left(\left|u_{2}\right| u_{2}-\left|u_{1}\right|^{3} u_{1}\right) H^{*} v_{\varepsilon} d \mu \\
& \leq \int_{\Omega_{0} \backslash \Omega_{\varepsilon}}\left(u_{2}-u_{1}\right) P\left(\left|u_{2}\right|,\left|u_{1}\right|\right) H^{*} v_{\varepsilon} d \mu \\
& \leq \varepsilon \int_{\Lambda \cup \Sigma} v_{\varepsilon} H\left(\chi_{\Omega_{0} \backslash \Omega_{\varepsilon}} P\left(\left|u_{2}\right|,\left|u_{1}\right|\right)\right) d \mu \\
& \leq \varepsilon\left\|v_{\varepsilon}\right\|_{L_{\mu}^{5}} f_{\varepsilon},
\end{aligned}
$$

where $\chi_{\Omega}$ denotes the characteristic function of the set $\Omega$ and

$$
f_{\varepsilon}=\left\|H\left(\chi_{\Omega_{0} \backslash \backslash \Omega_{\varepsilon}} P\left(\left|u_{2}\right|,\left|u_{1}\right|\right)\right)\right\|_{L_{\mu}^{5 / 4}} \rightarrow 0 \quad \text { as } \varepsilon \rightarrow 0 .
$$

To summarize,

$$
\int_{\Omega} a_{i j} \partial_{i} v_{\varepsilon} \partial_{j} v_{\varepsilon} d x \leq \varepsilon\left\|v_{\varepsilon}\right\|_{L_{\mu}^{5}} f_{\varepsilon}-g_{\varepsilon}
$$

where

$$
g_{\varepsilon}=\int_{\Gamma} \zeta\left(\left|u_{2}\right|^{p-1} u_{2}-\left|u_{1}\right|^{p-1} u_{1}\right) v_{\varepsilon} d s+\int_{\Omega_{\varepsilon}}\left(\left|u_{2}\right|^{3} u_{2}-\left|u_{1}\right|^{3} u_{1}\right) G^{*} v_{\varepsilon} d \mu .
$$

2. Derive the estimate (5.2): Note that $G^{*} v_{\varepsilon}=\varepsilon-H^{*} v_{\varepsilon} \geq \varepsilon-H^{*} \varepsilon \geq 0$ in $\Omega_{\varepsilon}$. Furthermore,

$$
\begin{aligned}
\int_{\Lambda \cup \Sigma} G v_{\varepsilon}^{4} v_{\varepsilon} d \mu & \leq \int_{\Omega_{0} \backslash \Omega_{\varepsilon}} v_{\varepsilon}^{4}\left|G^{*} v_{\varepsilon}\right| d \mu+\int_{\Omega_{\varepsilon}} v_{\varepsilon}^{4} G^{*} v_{\varepsilon} d \mu \\
& \leq \varepsilon^{5 / 2} \int_{\Omega_{0} \backslash \Omega_{\varepsilon}} v_{\varepsilon}^{3 / 2}\left|G^{*} v_{\varepsilon}\right| d \mu+\varepsilon^{4} \int_{\Omega_{\varepsilon}} G^{*} v_{\varepsilon} d \mu \\
& \leq \varepsilon^{5 / 2}\left\|v_{\varepsilon}^{3 / 2}\right\|_{L_{\mu}^{10 / 3}}\left\|G^{*} v_{\varepsilon}\right\|_{L_{\mu}^{5}}\left\|\chi_{\Omega_{0} \backslash \Omega_{\varepsilon}}\right\|_{L_{\mu}^{2}}+\varepsilon^{4} \int_{\Omega_{\varepsilon}} G^{*} v_{\varepsilon} d \mu \\
& \leq \varepsilon^{5 / 2}\left\|v_{\varepsilon}\right\|_{L_{\mu}^{5}}^{5 / 2} f_{\varepsilon}+\varepsilon^{4} \int_{\Omega_{\varepsilon}} G^{*} v_{\varepsilon} d \mu
\end{aligned}
$$

where $f_{\varepsilon}=2\left\|\chi_{\Omega_{0} \backslash \Omega_{\varepsilon}}\right\|_{L_{\mu}^{2}} \rightarrow 0$ as $\varepsilon \rightarrow 0$. To conclude,

$$
\left(\int_{\Gamma} \zeta\left|v_{\varepsilon}\right|^{p+1} d s\right)^{\frac{2}{p+1}}+\left(\int_{\Lambda \cup \Sigma}\left(G v_{\varepsilon}^{4}\right) v_{\varepsilon} d \mu\right)^{\frac{2}{5}} \leq \varepsilon\left\|v_{\varepsilon}\right\|_{L_{\mu}^{5}} f_{\varepsilon}+h_{\varepsilon}
$$

with

$$
h_{\varepsilon}=\left(\int_{\Gamma} \zeta\left|v_{\varepsilon}\right|^{p+1} d s\right)^{\frac{2}{p+1}}+\left(\varepsilon^{4} \int_{\Omega_{\varepsilon}} G^{*} v_{\varepsilon} d \mu\right)^{\frac{2}{5}} .
$$

3. Show that $h_{\varepsilon}$ can be compensated by $g_{\varepsilon}$ : We consider first the radiation terms. If $\int_{\Omega_{\varepsilon}} \varepsilon^{-1} G^{*} v_{\varepsilon} d \mu \rightarrow 0$ as $\varepsilon \rightarrow 0$, then

$$
\begin{gathered}
\left(\varepsilon^{4} \int_{\Omega_{\varepsilon}} G^{*} v_{\varepsilon} d \mu\right)^{\frac{2}{5}}-\int_{\Omega_{\varepsilon}}\left(\left|u_{2}\right|^{3} u_{2}-\left|u_{1}\right|^{3} u_{1}\right) G^{*} v_{\varepsilon} d \mu \\
\leq \varepsilon^{2}\left(\int_{\Omega_{\varepsilon}} \varepsilon^{-1} G^{*} v_{\varepsilon} d \mu\right)^{\frac{2}{5}} \leq \varepsilon\left\|v_{\varepsilon}\right\|_{L_{\mu}^{5}} f_{\varepsilon},
\end{gathered}
$$


where $f_{\varepsilon} \rightarrow 0$ as $\varepsilon \rightarrow 0$. Thus, we may assume $\int_{\Omega_{\varepsilon}} \varepsilon^{-1} G^{*} v_{\varepsilon} d \mu \rightarrow C_{1}>0$. Then, since $\varepsilon^{-1} G^{*} v_{\varepsilon} \rightarrow G^{*} 1$ in $\Omega_{0}$, there is $\varepsilon_{1}>0$ such that $\int_{\Omega_{\varepsilon_{1}}} G^{*} 1 d \mu$ is strictly positive. Now, we claim that there is $\varepsilon_{0}>0$ such that, for all $\varepsilon \leq \varepsilon_{0}$,

$$
\left(\varepsilon^{4} \int_{\Omega_{\varepsilon}} G^{*} v_{\varepsilon} d \mu\right)^{\frac{2}{5}}-\int_{\Omega_{\varepsilon}}\left(\left|u_{2}\right|^{3} u_{2}-\left|u_{1}\right|^{3} u_{1}\right) G^{*} v_{\varepsilon} d \mu \leq 0 .
$$

If (5.12) is not true, we may choose arbitrarily small $\varepsilon$ such that

$$
\begin{aligned}
\varepsilon\left(\int_{\Omega_{\varepsilon}} \varepsilon^{-1} G^{*} v_{\varepsilon} d \mu\right)^{\frac{2}{5}} & >\int_{\Omega_{\varepsilon}}\left(\left|u_{2}\right|^{3} u_{2}-\left|u_{1}\right|^{3} u_{1}\right) \varepsilon^{-1} G^{*} v_{\varepsilon} d \mu \\
& \geq \int_{\Omega_{\varepsilon_{1}}}\left(\left|u_{2}\right|^{3} u_{2}-\left|u_{1}\right|^{3} u_{1}\right) \varepsilon^{-1} G^{*} v_{\varepsilon} d \mu \\
& \geq C_{2} \varepsilon_{1} \int_{\Omega_{\varepsilon_{1}}} \varepsilon^{-1} G^{*} v_{\varepsilon} d \mu \\
& \geq C_{2} \varepsilon_{1} \int_{\Omega_{\varepsilon_{1}}} G^{*} 1 d \mu=C_{3}>0
\end{aligned}
$$

which is a contradiction. Using similar arguments, we see that

$$
\left(\int_{\Gamma} \zeta\left|v_{\varepsilon}\right|^{p+1} d s\right)^{\frac{2}{p+1}}-\int_{\Gamma}\left(\left|u_{2}\right|^{p-1} u_{2}-\left|u_{1}\right|^{p-1} u_{1}\right) v_{\varepsilon} d s \leq \varepsilon\left\|v_{\varepsilon}\right\|_{L_{\mu}^{5}} f_{\varepsilon},
$$

when $\varepsilon$ is small enough.

4. Show that $\mu\left(\Omega_{0}\right)+\mathcal{L}\left(\Omega_{0}\right)+\gamma\left(\Omega_{0}\right)=0$ : The steps $1-3$ imply that

$$
\left\|v_{\varepsilon}\right\|_{L_{\mu}^{5}} \leq \varepsilon f_{\varepsilon}
$$

when $\varepsilon$ is small enough. Hence

$$
\mu\left(\Omega_{\varepsilon}\right)=\varepsilon^{-1}\left(\int_{\Omega_{\varepsilon}} \varepsilon^{5} d \mu\right)^{1 / 5} \leq \varepsilon^{-1}\left\|v_{\varepsilon}\right\|_{L_{\mu}^{5}} \leq f_{\varepsilon} \rightarrow 0 .
$$

This is a contradiction, since also $\mu\left(\Omega_{\varepsilon}\right) \rightarrow \mu\left(\Omega_{0}\right)>0$. Therefore, $\mu\left(\Omega_{0}\right)=0$. From this fact it is straightforward to deduce $\mathcal{L}\left(\Omega_{0}\right)=\gamma\left(\Omega_{0}\right)=0$.

6. Boundedness of solutions. The following result is useful, for example, in analysing linearised conductive-radiative equations. Let us stress that if the source term $f$ does not depend on the radiative coefficients, then the bound $M$ given by Theorem 5 is independent of the radiative properties of $\Lambda \cup \Sigma$. We recall that $\phi$ and $\psi$ are called suband supersolutions of (2.2), respectively, if

$$
\begin{aligned}
& \langle Q \phi, v\rangle \leq\langle f, v\rangle \quad \forall v \in V^{+}, \\
& \langle Q \psi, v\rangle \geq\langle f, v\rangle \quad \forall v \in V^{+},
\end{aligned}
$$

where $V^{+}=\{v \in V: v \geq 0\}$.

TheOREm 5. Suppose that the right-hand side of (2.2) satisfies

$$
\langle f, v\rangle=\int_{\Omega} f_{1} v d x+\int_{\Sigma \cup \partial \Omega} f_{2} v d s
$$


with $f_{1} \in L^{p_{1}}(\Omega)$ and $f_{2} \in L^{p_{2}}(\Sigma \cup \partial \Omega)$ for $p_{1}>3 / 2$ and $p_{2}>2$. Then there exists a bound $M$ such that

(i) if $u$ is a subsolution, then

$$
\left\|u^{+}\right\|_{L^{\infty}(\Omega)}+\left\|u^{+}\right\|_{L^{\infty}(\Omega \cup \partial \Omega)} \leq M\left(\left\|u^{+}\right\|_{H^{1}(\Omega)}, f\right) ;
$$

(ii) if $u$ is a supersolution, then

$$
\left\|u^{-}\right\|_{L^{\infty}(\Omega)}+\left\|u^{-}\right\|_{L^{\infty}(\Sigma \cup \partial \Omega)} \leq M\left(\left\|u^{-}\right\|_{H^{1}(\Omega)}, f\right) ;
$$

(iii) if $u$ is a solution of $(2.2)$, then

$$
\|u\|_{L^{\infty}(\Omega)}+\|u\|_{L^{\infty}(\Sigma \cup \partial \Omega)} \leq M\left(\|u\|_{H^{1}(\Omega)}, f\right) .
$$

Proof. We use the technique of Moser iteration; see Gilbarg and Trudinger [6]. Assume first that $u$ is a subsolution. Let $\alpha \geq 1$ and define $B: \mathbb{R}_{+} \rightarrow \mathbb{R}_{+}$by $B(t)=t^{\alpha}$. Set $u_{k}=\min \left\{u^{+}, k\right\}, k>0$, and define

$$
\Phi\left(u_{k}\right)=\int_{0}^{u_{k}}\left(B^{\prime}(t)\right)^{2} d t=\frac{\alpha^{2}}{2 \alpha-1} u_{k}^{2 \alpha-1} .
$$

Clearly for fixed $k, \Phi\left(u_{k}\right) \in V^{+}$.

We claim first that

$$
\int_{\Gamma}|u|^{p} u \Phi\left(u_{k}\right) d s+\int_{\Lambda \cup \Sigma}\left(G|u|^{3} u\right) \Phi\left(u_{k}\right) d \mu \geq 0 .
$$

The term on $\Gamma$ is clearly positive. Note that

$$
\left\langle G|u|^{3} u, \Phi\left(u_{k}\right)\right\rangle_{\mu}=\left\langle G u_{+}^{4}, \Phi\left(u_{k}\right)\right\rangle_{\mu}-\left\langle G u_{-}^{4}, \Phi\left(u_{k}\right)\right\rangle_{\mu},
$$

where

$$
-\left\langle G u_{-}^{4}, \Phi\left(u_{k}\right)\right\rangle_{\mu}=-\left\langle u_{-}^{4}, \Phi\left(u_{k}\right)\right\rangle_{\mu}+\left\langle H u_{-}^{4}, \Phi\left(u_{k}\right)\right\rangle_{\mu} \geq 0
$$

since $u_{-}^{4} \Phi\left(u_{k}\right)=0$ and $H u_{-}^{4} \geq 0$. Furthermore,

$$
\left\langle G u_{+}^{4}, \Phi\left(u_{k}\right)\right\rangle_{\mu} \geq\left\langle G u_{+}^{4}, u_{k}^{2 \alpha-1}\right\rangle_{\mu}=\left\langle G u_{k}^{4}, u_{k}^{2 \alpha-1}\right\rangle_{\mu}+\left\langle G\left(u_{+}^{4}-u_{k}^{4}\right), u_{k}^{2 \alpha-1}\right\rangle_{\mu} .
$$

Denote next $r=(2 \alpha+3) / 4$ and $s=(2 \alpha+3) /(2 \alpha-1)$. Then

$$
\begin{aligned}
\left\langle G u_{k}^{4}, u_{k}^{2 \alpha-1}\right\rangle_{\mu} & \geq\left(\left\|u_{k}\right\|_{L_{\mu}^{4 r}}^{4 r}-\left\|H u_{k}^{4}\right\|_{L_{\mu}^{r}}\left\|u_{k}^{2 \alpha-1}\right\|_{L_{\mu}^{s}}\right) \\
& \geq\left(\left\|u_{k}\right\|_{L_{\mu}^{4 r}}^{4 r}-\|H\|_{L_{\mu}^{r}}\left\|u_{k}\right\|_{L_{\mu}^{4 r}}^{4}\left\|u_{k}\right\|_{L_{\mu}^{4 r}}^{4 r-4}\right) \geq 0 .
\end{aligned}
$$

Finally, since

$$
\begin{aligned}
u_{+}^{4}-u_{k}^{4} & =\left(u_{+}^{4}-k^{4}\right)^{+}, \\
u_{k}^{2 \alpha-1} & =-\left(u_{+}^{2 \alpha-1}-k^{2 \alpha-1}\right)^{-}+k^{2 \alpha-1},
\end{aligned}
$$

we have

$$
\begin{aligned}
\left\langle G\left(u_{+}^{4}-u_{k}^{4}\right), u_{k}^{2 \alpha-1}\right\rangle_{\mu}= & \left\langle u_{+}^{4}-u_{k}^{4}, G k^{2 \alpha-1}\right\rangle_{\mu} \\
& -\left\langle\left(u_{+}^{4}-k^{4}\right)^{+},\left(u_{+}^{2 \alpha-1}-u_{k}^{2 \alpha-1}\right)^{-}\right\rangle_{\mu} \\
& +\left\langle H\left(u_{+}^{4}-k^{4}\right)^{+},\left(u_{+}^{2 \alpha-1}-u_{k}^{2 \alpha-1}\right)^{-}\right\rangle_{\mu} \geq 0
\end{aligned}
$$


The first term above is positive since $G 1 \geq 0$. In the second term the product of the positive and negative part is zero and the positivity of the third term follows from the positivity of $H$. Accordingly, (6.1) holds.

The rest of the proof is fairly standard. Using estimate (6.1), we find that

$$
\int_{\Omega} a_{i j} \partial_{i} u \partial_{j} \Phi\left(u_{k}\right) d x \leq\left\langle f, \Phi\left(u_{k}\right)\right\rangle
$$

since $u$ is a subsolution. Moreover,

$$
\int_{\Omega} a_{i j} \partial_{i} u \partial_{j} \Phi\left(u_{k}\right) d x=\int_{\Omega} a_{i j} \partial_{i} u_{k} \partial_{j} u_{k} B^{\prime}\left(u_{k}\right)^{2} d x \geq C \int_{\Omega}\left|\nabla B\left(u_{k}\right)\right|^{2} d x
$$

since the operator $A$ is strictly elliptic and $\nabla \Phi\left(u_{k}\right)=0$ when $u \neq u_{k}$. Denote next $\widetilde{\Sigma}=\Sigma \cup \partial \Omega$. Since $B\left(u_{k}\right) \in H^{1}(\Omega)$, we have

$$
\begin{aligned}
\left\|B\left(u_{k}\right)\right\|_{L^{6}(\Omega)}^{2}+\left\|B\left(u_{k}\right)\right\|_{L^{4}(\tilde{\Sigma})}^{2} & \leq C_{1}\left(\int_{\Omega}\left|\nabla B\left(u_{k}\right)\right|^{2} d x+\int_{\Omega}\left|B\left(u_{k}\right)\right|^{2} d s\right) \\
& \leq C_{2}\left(\left\langle f, \Phi\left(u_{k}\right)\right\rangle+\int_{\Omega}\left|B\left(u_{k}\right)\right|^{2} d x\right),
\end{aligned}
$$

where $C_{2}$ does not depend on $\alpha$ or $k$. Set next $p^{*}=\min \left\{p_{1}, 6\right\}$ and choose $q_{1}$ and $q_{2}$ such that $1 / p^{*}+1 / q_{1}=1,1 / p_{2}+1 / q_{2}=1$. Then

$$
\begin{aligned}
\int_{\Omega} f_{1} \Phi\left(u_{k}\right)+\left|B\left(u_{k}\right)\right|^{2} d x & \leq \alpha^{2} \int_{\Omega}\left(f_{1}+u^{+}\right) u_{k}^{2 \alpha-1} d \eta \\
& \leq \alpha^{2}\left\|f_{1}+u^{+}\right\|_{L^{p^{*}}(\Omega)}\left\|u_{k}^{2 \alpha}\right\|_{L^{q_{1}}(\Omega)}
\end{aligned}
$$

where $\left\|u^{+}\right\|_{L^{p^{*}}(\Omega)} \leq C\left\|u^{+}\right\|_{H^{1}(\Omega)}$. Similarly, we see that

$$
\int_{\tilde{\Sigma}} f_{2} \Phi\left(u_{k}\right) d s \leq \alpha^{2} \int_{\widetilde{\Sigma}} f_{2} u_{k}^{2 \alpha-1} d s \leq \alpha^{2}\left\|f_{2}\right\|_{L^{p_{2}(\tilde{\Sigma})}}\left\|u_{k}^{2 \alpha}\right\|_{L^{q_{2}(\tilde{\Sigma})}}
$$

Now, from (6.3)-(6.5) we get

$$
\left\|u_{k}\right\|_{L^{6 \alpha}(\Omega)}^{2 \alpha}+\left\|u_{k}\right\|_{L^{4 \alpha}(\widetilde{\Sigma})}^{2 \alpha} \leq \alpha^{2} C_{4}\left(\left\|u_{k}\right\|_{L^{2 \alpha q_{1}(\Omega)}}^{2 \alpha}+\left\|u_{k}\right\|_{L^{2 \alpha q_{2}(\tilde{\Sigma})}}^{2 \alpha}\right) .
$$

Since $C_{4}$ does not depend on $k$, we can set $k \rightarrow \infty$, so that the above inequality holds for $u_{+}$. Set next $r=2 q_{1}$ and $s=2 q_{2}$ and choose $\chi=\min \{6 / r, 4 / s\}>1$. Then

$$
\left\|u_{+}\right\|_{L^{r \chi \alpha}(\Omega)} \leq C(\Omega)\left\|u_{+}\right\|_{L^{6 \alpha}(\Omega)}, \quad\left\|u_{+}\right\|_{L^{s \chi \alpha}(\widetilde{\Sigma})} \leq C(\widetilde{\Sigma})\left\|u_{+}\right\|_{L^{4 \alpha}(\tilde{\Sigma})},
$$

and thus

$$
\left\|u_{+}\right\|_{L^{r \chi \alpha}(\Omega)}+\left\|u_{+}\right\|_{L^{s \chi \alpha}(\tilde{\Sigma})} \leq\left(\alpha C_{5}\right)^{1 / \alpha}\left(\left\|u_{+}\right\|_{L^{r \alpha}(\Omega)}+\left\|u_{+}\right\|_{L^{s \alpha}(\tilde{\Sigma})}\right) .
$$

$L^{\infty}$-estimates of $u^{+}$now follow by iteration of (6.7). Namely, we take $\alpha=\chi^{m}, m=$ $0,1,2, \ldots$, so that

$$
\begin{aligned}
\left\|u_{+}\right\|_{L^{r \chi^{m+1}}(\Omega)}+\left\|u_{+}\right\|_{L^{s \chi} \chi^{m+1}(\tilde{\Sigma})} & \leq\left(C_{5} \chi^{m}\right)^{1 / \chi^{m}}\left(\left\|u_{+}\right\|_{L^{r \chi^{m}}(\Omega)}+\left\|u_{+}\right\|_{L^{s \chi^{m}}(\tilde{\Sigma})}\right) \\
& \leq C_{5}^{\zeta} \chi^{\eta}\left(\left\|u_{+}\right\|_{L^{r}(\Omega)}+\left\|u_{+}\right\|_{L^{s}(\tilde{\Sigma})}\right)
\end{aligned}
$$

where

$$
\zeta=\sum_{n=0}^{m} \chi^{-n}, \quad \eta=\sum_{n=0}^{m} n \chi^{-n} .
$$


Therefore, the claim (i) follows by letting $m \rightarrow \infty$. The claims (ii) and (iii) follow by replacing $u$ with $-u$.

7. Time dependence. Radiation propagates at the speed of light and, hence, the radiative transfer is instantaneous compared to heat conduction and convection. Consequently, we may formulate the time-dependent conductive-radiative problem as: for $f \in X^{*}$ and $u_{0} \in L^{2}(\Omega)$, we seek $u \in W$ such that

$$
\begin{aligned}
\left\langle u^{\prime}(t) . v\right\rangle_{V}+\langle Q u(t), v\rangle_{V} & =\langle f(t), v\rangle_{V}, \\
u(0) & =u_{0},
\end{aligned}
$$

for all $v \in V$ and almost all $t \in[0, T]$. For simplicity we have set heat capacity equal to one and chosen $p=1$ in the definition of the local heat flux term on $\Gamma$.

This problem is nontrivial since for the Galerkin method we need an a priori estimate in the space $L^{5}\left(0, T ; L_{\mu}^{5}\right)$ which, in general, does not follow from the coercivity of the stationary operator $Q: V \rightarrow V^{*}$ (see Theorem 3). Metzger [12] solved this problem by assuming additionally that $\|H\|<1$, which restricts the analysis to radiative systems without enclosures. Here we adopt a different point of view by assuming slightly more regularity from the data, which allows us to derive the desired a priori estimate via Moser iteration.

Our idea is to solve first an auxiliary problem for which the a priori estimate in $L^{5}\left(0, T ; L_{\mu}^{5}\right)$ trivially holds: fix $\varepsilon>0$ and seek $u_{\varepsilon}$ such that

$$
\begin{aligned}
\left\langle u_{\varepsilon}^{\prime}(t), v\right\rangle_{V}+\left\langle Q u_{\varepsilon}(t), v\right\rangle_{V}+\varepsilon \int\left|u_{\varepsilon}(t)\right|^{3} u_{\varepsilon}(t) v d \mu & =\langle f(t), v\rangle_{V}, \\
u(0) & =u_{0},
\end{aligned}
$$

for all $v \in V$ and almost all $t \in(0, T)$. Then, we will prove with Moser-type arguments that the auxiliary problem is in $L^{5}\left(0, T ; L_{\mu}^{5}\right)$ independently of $\varepsilon>0$, which allows us to deduce that $u_{\varepsilon}$ converges to a solution of the original problem. We note that if $\|H\|<1$, then the existence of solutions without any additional regularity assumptions follows from the analysis of the auxiliary problem.

Let us introduce first some notation and outline the basic properties of the function spaces $X, X^{*}$ and $W$. Throughout this section we shall use the following abbreviations:

$$
\langle u, v\rangle=\langle u, v\rangle_{V}, \quad(u, v)=(u, v)_{L^{2}(\Omega)}, \quad L^{p}(B)=L^{p}(0, T ; B) .
$$

We equip the space $X$ with the norm

$$
\|u\|_{X}=\|u\|_{L^{2}\left(H^{1}(\Omega)\right)}+\|u\|_{L^{5}\left(L_{\mu}^{5}\right)} .
$$

To define the norm for $X^{*}$, we note that each $w \in X^{*}$ can be written as $w=u+v$ with $u \in L^{2}\left(H^{1}(\Omega)^{*}\right)$ and $v \in L^{5 / 4}\left(L_{\mu}^{5 / 4}\right)$; see [5]. Therefore, we can define

$$
\|w\|_{X^{*}}=\inf _{w=u+v}\left\{\|u\|_{\left.L^{2}\left(H^{1}(\Omega)\right)^{*}\right)}+\|v\|_{L^{5 / 4}\left(L_{\mu}^{5 / 4}\right)}\right\}
$$

and finally, we define the norm of $W$ as

$$
\|u\|_{W}=\|u\|_{X}+\left\|u^{\prime}\right\|_{X^{*}} .
$$


The most important properties of the space $W$ are summarized in the following lemma. Gajewski et al. [5, Chapter IV] analysed a similar space where a Hilbert space was used instead of $L_{\mu}^{5}$. Nevertheless, the proofs given in [5] apply also in this case, although the assertions there are not stated in the generality needed here.

Lemma 10. The spaces $X, X^{*}$, and $W$ are reflexive Banach spaces. Furthermore, the embedding $W \subset C\left([0, T] ; L^{2}(\Omega)\right)$ is continuous and the following integration by parts formula holds:

$$
(u(t), v(t))-(u(s), v(s))=\int_{s}^{t}\left\langle u^{\prime}(z), v(z)\right\rangle+\left\langle v^{\prime}(z), u(z)\right\rangle d z
$$

for all $u, v \in W$ and $s, t \in[0, T], s \leq t$.

The most important properties of the stationary operator $Q: V \rightarrow V^{*}$ readily extend to the time-dependent case.

Lemma 11. The operator $Q: X \rightarrow X^{*}$ is bounded and pseudomonotone.

Proof. From the boundedness of $A$ and $G$ together with the Hölder inequality, it follows that there is a constant $C>0$ such that

$$
\|Q u\|_{X^{*}} \leq C\left\{\|u\|_{L^{2}\left(H^{1}(\Omega)\right)}+\|u\|_{L^{5}\left(L_{\mu}^{5}\right)}^{4}\right\} \quad \forall u \in X .
$$

Further, $A: X \rightarrow X^{*}$ is monotone because $A: V \rightarrow V^{*}$ is monotone.

To prove pseudomonotonicity of the radiation part, let $u_{i} \rightarrow u$ weakly in $X$. Then $\left|u_{i}\right|^{3} u_{i} \rightarrow|u|^{3} u$ weakly in $L^{5 / 4}\left(0, T ; L_{\mu}^{5 / 4}\right)$, by the arguments of Lemma 7 . Hence

$$
\lim _{i \rightarrow \infty} \int_{0}^{T}\left\langle G\left|u_{i}\right|^{3} u_{i}, v\right\rangle_{\mu} d t=\int_{0}^{T}\left\langle G|u|^{3} u, v\right\rangle_{\mu} d t \quad \forall v \in X .
$$

Thus, Fatou's lemma implies

$$
\begin{aligned}
\underline{\lim }_{i \rightarrow \infty}\left\langle G\left|u_{i}\right|^{3} u_{i}, u_{i}-v\right\rangle_{X} \\
\quad \geq \underline{\lim }_{i \rightarrow \infty} \int_{0}^{T}\left\langle G\left|u_{i}\right|^{3} u_{i}, u_{i}\right\rangle_{\mu} d t-\varlimsup_{i \rightarrow \infty} \int_{0}^{T}\left\langle G\left|u_{i}\right|^{3} u_{i}, v\right\rangle_{\mu} d t \\
\quad \geq \int_{0}^{T} \underline{\lim }_{i \rightarrow \infty}\left\langle G\left|u_{i}\right|^{3} u_{i}, u_{i}\right\rangle_{\mu} d t-\lim _{i \rightarrow \infty} \int_{0}^{T}\left\langle G\left|u_{i}\right|^{3} u_{i}, v\right\rangle_{\mu} d t \\
\geq \int_{0}^{T}\left\langle G|u|^{3} u, u\right\rangle_{\mu} d t-\int_{0}^{T}\left\langle G|u|^{3} u, v\right\rangle_{\mu} d t
\end{aligned}
$$

where we used the pseudomonotonicity of $G|u|^{3} u: V \rightarrow V^{*}$.

7.1. Auxiliary problem. To introduce the Galerkin equation for the auxiliary problem (7.3)-(7.4), let $\left\{w_{1}, w_{2}, \ldots\right\}$ be a basis in $V$, define $V_{n}=\operatorname{span}\left\{w_{1}, \ldots, w_{n}\right\}$ and set

$$
u_{n}(t)=\sum_{k=1}^{n} c_{k n}(t) w_{k}
$$


Then, we seek $u_{n} \in W$ such that

$$
\begin{aligned}
\left\langle u_{n}^{\prime}(t), w_{j}\right\rangle+\left\langle Q u_{n}(t), w_{j}\right\rangle+\varepsilon \int\left|u_{n}(t)\right|^{3} u_{n}(t) w_{j} d \mu & =\left\langle f(t), w_{j}\right\rangle, \\
u_{n}(0) & =u_{n 0} \in V_{n},
\end{aligned}
$$

for $j=1, \ldots, n$, where $u_{n 0}$ is chosen such that $u_{n 0} \rightarrow u_{0}$ in $L^{2}(\Omega)$ as $n \rightarrow \infty$.

Lemma 12. Let $u_{0} \in L^{2}(\Omega), f \in X^{*}$ and suppose $u_{n}$ is a solution of (7.6)-(7.7). Then there exists a constant $C>0$, independent of $n$, such that

$$
\begin{aligned}
\left\|u_{n}\right\|_{X} & \leq C, \\
\left\|Q u_{n}\right\|_{X^{*}} & \leq C, \\
\max _{0 \leq t \leq T}\left\|u_{n}(t)\right\|_{L^{2}(\Omega)} & \leq C .
\end{aligned}
$$

Proof. We multiply (7.6) by $c_{j n}(t)$ and sum for $j=1, \ldots, n$, so that

$$
\left\langle u_{n}^{\prime}, u_{n}\right\rangle+\left\langle Q u_{n}, u_{n}\right\rangle+\varepsilon \int\left|u_{n}\right|^{5} d \mu=\left\langle f, u_{n}\right\rangle .
$$

Then, since

$$
\left\langle u_{n}^{\prime}, u_{n}\right\rangle=\left(u_{n}^{\prime}, u_{n}\right)=\frac{d}{d t}\left(\frac{1}{2}\left\|u_{n}\right\|_{L^{2}(\Omega)}^{2}\right),
$$

integration of (7.11) from 0 to $t$ gives

$$
\begin{aligned}
\left\|u_{n}(t)\right\|_{L^{2}(\Omega)}^{2}+2 \int_{0}^{t}\left\langle Q u_{n}, u_{n}\right\rangle d s+2 \varepsilon \int_{0}^{t}\left\|u_{n}\right\|_{L_{\mu}^{5}}^{5} d s & \\
& =\left\|u_{n}(0)\right\|_{L^{2}(\Omega)}^{2}+2 \int_{0}^{t}\left\langle f, u_{n}\right\rangle d s .
\end{aligned}
$$

Therefore, (7.10) is valid provided (7.8) holds.

By Theorem 3 , there is a constant $\theta>0$ such that

$$
2 \int_{0}^{T}\left\langle Q u_{n}, u_{n}\right\rangle d s \geq 2 \int_{0}^{T} \theta\left(\left\|u_{n}\right\|_{V}^{2}-1\right) d s \geq 2 \theta\left\|u_{n}\right\|_{L^{2}\left(H^{1}(\Omega)\right)}^{2}-2 \theta T .
$$

Furthermore, applying the inequality

$$
a b \leq \lambda \frac{a^{p}}{p}+\lambda^{-q / p} \frac{b^{q}}{q}, \quad \frac{1}{p}+\frac{1}{q}=1, \quad a, b \geq 0, \quad \lambda>0,
$$

and writing $f=f_{1}+f_{2}, f_{1} \in L^{2}\left(H^{1}(\Omega)^{*}\right), f_{2} \in L^{5 / 4}\left(L_{\mu}^{5 / 4}\right)$, we have

$$
\begin{aligned}
2 \int_{0}^{T}\left\langle f, u_{n}\right\rangle d s & =2 \int_{0}^{T}\left\langle f_{1}, u_{n}\right\rangle_{H^{1}(\Omega)} d s+2 \int_{0}^{T}\left\langle f_{2}, u_{n}\right\rangle_{\mu} d s \\
& \leq 2\left\|u_{n}\right\|_{L^{2}\left(H^{1}(\Omega)\right)}\left\|f_{1}\right\|_{L^{2}\left(H^{1}(\Omega)^{*}\right)}+2\left\|u_{n}\right\|_{L^{5}\left(L_{\mu}^{5}\right)}\left\|f_{2}\right\|_{L^{5 / 4}\left(L_{\mu}^{5 / 4}\right)} \\
& \leq \theta\left\|u_{n}\right\|_{L^{2}\left(H^{1}(\Omega)\right)}^{2}+C(\theta)\left\|f_{1}\right\|_{L^{2}\left(H^{1}(\Omega)^{*}\right)}^{2}+C(\varepsilon)\left\|f_{2}\right\|_{L^{5 / 4}\left(L_{\mu}^{5 / 4}\right)}^{5 / 4}
\end{aligned}
$$

Hence, collecting estimates (7.12), (7.13), and (7.14), we arrive at (7.8). Using the boundedness of $Q$, the estimate (7.10) follows at once from (7.8).

Lemma 13. There exists a solution for the auxiliary problem (7.3)-(7.4). 
Proof. For simplicity, we shall denote the solution candidate by $u$ instead of $u_{\varepsilon}$.

1. Existence of solutions for Galerkin equation (7.6)-(7.7). The Galerkin equation can be interpreted as a system of ordinary differential equations, and, hence, the existence of solutions can be deduced from the theorem of Carathéodory [23]. To apply this theorem, we need to note the following:

(i) If $v(t)$ is a solution of (7.6)-(7.7), then $\|v(t)\|_{L^{2}(\Omega)} \leq C$ by (7.10).

(ii) The mapping $t \mapsto\left\langle Q v, w_{j}\right\rangle$ is measurable on $(0, T)$ and for all $v \in V_{n}$.

(iii) The mapping $v \mapsto\left\langle Q v, w_{j}\right\rangle$ is continuous on $V_{n}$.

Let $\left\{u_{n}\right\}$ be a sequence of solutions of (7.6)-(7.7). Then, according to Lemma 12, there exist $u \in X, w \in X^{*}$, and $z \in L^{2}(\Omega)$ such that $u_{n} \rightarrow u$ weakly in $X, Q u_{n} \rightarrow w$ weakly in $X^{*}$, and $u_{n}(t) \rightarrow z$ weakly in $L^{2}(\Omega)$ as $n \rightarrow \infty$.

2. Show that

$$
(z, \psi(T) v)-\left(u_{0}, \psi(0) v\right)=\int_{0}^{T}\langle f(t)-w(t), \psi(t) v\rangle+\left\langle\psi^{\prime}(t) v, u(t)\right\rangle d t
$$

for all $\psi \in C^{\infty}[0, T]$ and $v \in V$. First, let $\psi(t) \in C^{\infty}[0, T]$ and $v \in V_{m}, m \leq n$. Then, integrating by parts,

$$
\begin{aligned}
\left(u_{n}(T), \psi(T) v\right)-\left(u_{n}(0), \psi(0) v\right) \\
\quad=\int_{0}^{T}\left\langle u_{n}^{\prime}(t), \psi(t) v\right\rangle+\left\langle\psi^{\prime}(t) v, u_{n}(t)\right\rangle d t \\
\quad=\int_{0}^{T}\left\langle f(t)-Q u_{n}(t), \psi(t) v\right\rangle+\left\langle\psi^{\prime}(t) v, u_{n}(t)\right\rangle d t \\
\quad=\left\langle f-Q u_{n}, \psi v\right\rangle_{X}+\left\langle\psi^{\prime} v, u_{n}\right\rangle_{X} .
\end{aligned}
$$

Thus, letting $n \rightarrow \infty$ we get

$$
(z, \psi(T) v)-\left(u_{0}, \psi(0) v\right)=\langle f-w, \psi v\rangle_{X}+\left\langle\psi^{\prime} v, u\right\rangle_{X} \quad \forall v \in \bigcup_{m} V_{m} .
$$

Because $\bigcup_{m} V_{m}$ is dense in $V$, this formula holds for all $v \in V$.

3. Prove next that $u, w, z$ satisfy

$$
\begin{aligned}
& u^{\prime}+w=f, \quad u \in W, \\
& u(0)=u_{0}, \quad u(T)=z .
\end{aligned}
$$

From (7.15) it follows that

$$
\int_{0}^{T}\langle f(t)-w(t), v\rangle \psi(t) d t=-\int_{0}^{T}\langle v, u(t)\rangle \psi^{\prime}(t) d t
$$

for all $\psi \in C^{\infty}(0, T)$. This means that $u^{\prime}$ exists and $u^{\prime}=f-w \in X^{*}$. Hence also $u \in W$. Furthermore, integration by parts gives

$$
(u(T), \psi(T) v)-(u(0), \psi(0) v)=\int_{0}^{T}\left\langle u^{\prime}(t), \psi(t) v\right\rangle+\left\langle\psi^{\prime}(t) v, u(t)\right\rangle d t
$$

for all $\psi \in C^{\infty}[0, T], v \in V$. Therefore, (7.15) and (7.19) imply

$$
(u(T), \psi(T) v)-(u(0), \psi(0) v)=(z, \psi(T) v)-\left(u_{0}, \psi(0) v\right),
$$

so that $u(T)=z$ and $u(0)=u_{0}$. 
4. Finally, prove that $Q u=w$. Since $Q: X \rightarrow X^{*}$ is pseudomonotone, it satisfies the so-called condition (M); see Zeidler [23, Ch. 27]. This means that the weak convergence of $u_{n}$ and $Q u_{n}$ together with

$$
\varlimsup_{n \rightarrow \infty}\left\langle Q u_{n}, u_{n}\right\rangle \leq\langle w, u\rangle
$$

imply that $Q u=w$.

Integrating by parts we have

$$
\begin{aligned}
\frac{1}{2}\left(\left\|u_{n}(T)\right\|_{L^{2}(\Omega)}^{2}-\left\|u_{n}(0)\right\|_{L^{2}(\Omega)}^{2}\right) & =\int_{0}^{T}\left\langle u_{n}^{\prime}(t), u_{n}(t)\right\rangle d t \\
& =\int_{0}^{T}\left\langle f-Q u_{n}, u_{n}\right\rangle d t
\end{aligned}
$$

or

$$
\left\langle Q u_{n}, u_{n}\right\rangle_{X}=\left\langle f, u_{n}\right\rangle_{X}+\frac{1}{2}\left(\left\|u_{n}(0)\right\|_{L^{2}(\Omega)}^{2}-\left\|u_{n}(T)\right\|_{L^{2}(\Omega)}^{2}\right) .
$$

Therefore,

$$
\varlimsup_{n \rightarrow \infty}\left\langle Q u_{n}, u_{n}\right\rangle \leq\langle f, u\rangle_{X}+\frac{1}{2}\left(\|u(0)\|_{L^{2}(\Omega)}^{2}-\|u(T)\|_{L^{2}(\Omega)}^{2}\right)
$$

as $u_{n}(0) \rightarrow u(0)$ and $\|u(T)\|_{L^{2}(\Omega)} \leq \underline{\lim }\left\|u_{n}(T)\right\|_{L^{2}(\Omega)}$. This implies (7.21) since

$$
\frac{1}{2}\left(\|u(0)\|_{L^{2}(\Omega)}^{2}-\|u(T)\|_{L^{2}(\Omega)}^{2}\right)=\int_{0}^{T}\left\langle u^{\prime}, u\right\rangle d t=\langle w-f, u\rangle_{X} .
$$

Consequently, $Q u=w$.

7.2. Existence of solutions for the original problem.

Lemma 14. Suppose $u_{0} \in L^{5}(\Omega)$ and assume that

$$
f(t) \in L^{2}((0, T) \times \Omega)+L^{5+\delta}\left(L^{5 / 3+\delta}(\Sigma \cup \Gamma)\right)
$$

for some fixed $\delta>0$. Then there exists a constant $C>0$, independent of $\varepsilon$, such that the solution of (7.3)-(7.4) satisfies $\left\|u_{\varepsilon}\right\|_{W} \leq C$.

Proof. Clearly $u_{\varepsilon}^{\prime}$ is bounded in $X^{*}$ provided that $u_{\varepsilon}$ is bounded in $X$. Furthermore, arguing as in the proof of Lemma 12 , we easily see that $\left\|u_{\varepsilon}\right\|_{L^{2}(V)} \leq C$ independent of $\varepsilon$. Hence, it suffices to prove that

$$
\int_{0}^{T}\left\|u_{\varepsilon}(t)\right\|_{L_{\mu}^{5}}^{5} d t \leq C \int_{0}^{T}\left\|u_{\varepsilon}(t)\right\|_{L^{5}(\Omega)}^{5}+\left\|u_{\varepsilon}(t)\right\|_{L^{5}(\Sigma)}^{5} d t
$$

is bounded uniformly in $\varepsilon$. Actually, we shall prove a stronger statement:

$$
\left\|u_{\varepsilon}\right\|_{L^{25 / 3}((0, T) \times \Omega)}+\left\|u_{\varepsilon}\right\|_{L^{5}\left(L^{10}(\Sigma \cup \Gamma)\right)} \leq C\left(f, u_{0},\|u\|_{L^{2}(V)}\right) .
$$

We use a Moser-type argument to derive a bound for $\left(u_{\varepsilon}\right)^{+}$; the proof for $\left(u_{\varepsilon}\right)^{-}$is similar. To keep the presentation simple, we henceforth denote $u_{\varepsilon}$ simply by $u$ and define $B(t), u_{k}$, and $\Phi\left(u_{k}\right)$ as in Theorem 5 .

Suppose next that $u$ is a subsolution of (7.3)-(7.4), i.e., $u(0)=u_{0}$ and

$$
\left\langle u^{\prime}(t), v\right\rangle+\langle Q u(t), v\rangle+\varepsilon \int|u(t)|^{3} u(t) v d \mu \leq\langle f(t), v\rangle \quad \forall v \geq 0, v \in V .
$$


Then, choosing $v=\Phi\left(u_{k}\right)$, we have

$$
\left\langle u^{\prime}, \Phi\left(u_{k}\right)\right\rangle+C\left\|B\left(u_{k}\right)\right\|_{L^{6}(\Omega)}^{2}+C\left\|B\left(u_{k}\right)\right\|_{L^{4}(\Sigma)}^{2} \leq\left\langle f, \Phi\left(u_{k}\right)\right\rangle+C\left\|B\left(u_{k}\right)\right\|_{L^{2}(\Omega)}^{2}
$$

as in Theorem 3. We integrate next from 0 to $t$ and send $k \rightarrow \infty$. Then

$$
\begin{aligned}
\int_{0}^{t}\left\langle f, \Phi\left(u_{k}\right)\right\rangle & +\left\|B\left(u_{k}\right)\right\|_{L^{2}(\Omega)}^{2} d t \\
& \rightarrow C(\alpha)\left(\int_{0}^{t} \int_{\Omega}\left(f+u_{+}\right) u_{+}^{2 \alpha-1} d x d t+\int_{0}^{t} \int_{\Sigma \cup \Gamma} f u_{+}^{2 \alpha-1} d x d t\right)
\end{aligned}
$$

and

$$
\begin{aligned}
\int_{0}^{t}\left\langle u^{\prime}, \Phi\left(u_{k}\right)\right\rangle d t & \rightarrow \int_{0}^{t}\left\langle u^{\prime}, \Phi\left(u_{+}\right)\right\rangle d t \\
& =C(\alpha) \int_{0}^{t} \int_{\Omega} \frac{d}{d t} u_{+}^{2 \alpha}(t) d x d t \\
& =C(\alpha)\left(\left\|u_{+}^{\alpha}(t)\right\|_{L^{2}(\Omega)}^{2}-\left\|\left(u_{0}\right)_{+}^{\alpha}\right\|_{L^{2}(\Omega)}^{2}\right)
\end{aligned}
$$

Hence, collecting the previous estimates,

$$
\begin{aligned}
& \left\|u_{+}^{\alpha}\right\|_{L^{\infty}\left(L^{2}(\Omega)\right)}^{2}+\left\|u_{+}^{\alpha}\right\|_{L^{2}\left(L^{6}(\Omega)\right)}^{2}+\left\|u_{+}^{\alpha}\right\|_{L^{2}\left(L^{4}(\Sigma \cup \Gamma)\right)}^{2} \\
& \leq C(\alpha)\left(C_{1}\left\|u_{+}^{2 \alpha-1}\right\|_{L^{q_{1}}\left(L^{q_{2}}(\Omega)\right)}+C_{2}\left\|u_{+}^{2 \alpha-1}\right\|_{L^{q_{3}}\left(L^{q_{4}}(\Sigma \cup \Gamma)\right)}+C_{3}\right)
\end{aligned}
$$

with

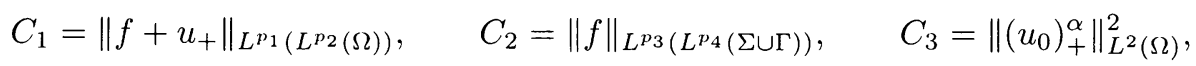

where the pairs of conjugate exponents $p_{i}, q_{i}, i=1, \ldots, 4$, are to be determined. We can further interpolate between the norms so that

$$
\|v\|_{L^{10 / 3}((0, T) \times \Omega)} \leq C\left(\|v\|_{L^{\infty}\left(L^{2}(\Omega)\right)}+\|v\|_{L^{2}\left(L^{6}(\Omega)\right)}\right)
$$

for all $v \in L^{\infty}\left(L^{2}(\Omega)\right) \cap L^{2}\left(L^{6}(\Omega)\right)$; see [1]. Now, the assertion follows by iteration of (7.25) if the constants $C_{i}$ are finite and the powers of $u^{+}$are greater on the left-hand side of (7.25) than on the right-hand side for all $1 \leq \alpha \leq 5 / 2$. Namely, we require

$$
10 / 3>q_{1}(2 \alpha-1), \quad 10 / 3>q_{2}(2 \alpha-1), \quad 2>q_{3}(2 \alpha-1), \quad 4>q_{4}(2 \alpha-1) .
$$

Clearly such $p_{i}, q_{i}$ can be found if $u_{0}$ and $f$ have assumed regularity. Note also that the terms on the right-hand side of (7.25) can be bounded by $\|u\|_{L^{2}(V)}$ when $\alpha=1$, and hence the bounding constant in (7.23) does not depend on $\varepsilon$.

REMARK 7.1. Lemma 14 could be slightly generalized. Namely, the iteration of (7.25) and hence the entire Lemma 14 are still valid if

$$
f(t) \in L^{1}\left(L^{5+\delta}(\Omega)\right)+L^{5+\delta}\left(L^{\frac{15}{11}+\delta}(\Omega)\right)+L^{5+\delta}\left(L^{5 / 3+\delta}(\Sigma \cup \partial \Omega)\right) .
$$

Nevertheless, the assumption (7.22) provides a reasonable compromise between generality and clarity of the proof.

Theorem 6. Suppose that either the hypotheses of Lemma 14 hold or $\|H\|<1$. Then there exists a solution for (7.1)-(7.2). 
Proof. Assume first the regularity required in Lemma 14. Then from the a priori estimate of Lemma 14 it follows that the sequence $\left\{u_{\varepsilon}\right\}$ is bounded in $W$. Hence there is $u \in W$ such that $u_{\varepsilon} \rightarrow u$ weakly in $W$ as $\epsilon \rightarrow 0$. Repeating the arguments of Lemma 13 , it is easy to see that $u$ solves $(7.1)-(7.2)$.

On the other hand, if $\|H\|<1$, then

$$
\langle Q u, u\rangle \geq C\left(\|u\|_{H^{1}(\Omega)}^{2}+\|u\|_{L_{\mu}^{5}}^{5}\right),
$$

which allows us to deduce the existence via the Galerkin method as in Subsection 7.1.

7.3. Uniqueness and comparison principle.

THEOREM 7. Let $u_{1}$ and $u_{2}$ be solutions of $(7.1)-(7.2)$ corresponding to the right-hand sides $f_{1}, f_{2} \in X^{*}$ and initial data $u_{0}^{1}, u_{0}^{2} \in L^{2}(\Omega)$ satisfying

$$
\begin{aligned}
&\left\langle f_{1}-f_{2}, w\right\rangle_{X} \geq 0 \forall w \geq 0, w \in W, \\
&\left(u_{0}^{1}-u_{0}^{2}, v\right) \geq 0 \quad \forall v \geq 0, v \in L^{2}(\Omega) .
\end{aligned}
$$

Then $u_{1} \geq u_{2}$ almost everywhere in $\Omega \times(0, T)$ with respect to measures $\mathcal{L}^{1} \times \mathcal{L}^{n}, \mathcal{L}^{1} \times \mu$, and $\mathcal{L}^{1} \times \gamma$. Consequently, the solution of (7.1)-(7.2), if it exists, is unique.

Proof. The proof is essentially the same as in the stationary case. The only difference is that instead of $\Omega_{0}$ defined in the proof of Theorem 4 , we are now interested in the set

$$
\left\{(x, t) \in \bar{\Omega} \times[0, T]: u_{1}(x, t)<u_{2}(x, t)\right\},
$$

which we claim to be of measure zero.

For any $t \in[0, T]$ we define $\Omega_{0}(t), \Omega_{\varepsilon}(t)$, and $v_{\varepsilon}(x, t)$ as in Sec. 5. Now, our aim is to prove that

$$
\begin{aligned}
\int_{0}^{T} \mu\left(\Omega_{\varepsilon}(t)\right) d t & =\int_{0}^{T} \int_{\Omega_{\varepsilon}(t)} d \mu d t \\
& =\int_{0}^{T} \varepsilon^{-1}\left(\int_{\Omega_{\varepsilon}(t)} \varepsilon^{5} d \mu\right)^{\frac{1}{5}} d t \\
& \leq \varepsilon^{-1} \int_{0}^{T}\left\|v_{\varepsilon}\right\|_{L_{\mu}^{5}} d t \\
& \leq \varepsilon^{-1} C(T)\left\|v_{\varepsilon}\right\|_{L^{2}\left(L_{\mu}^{5}\right)} \rightarrow 0 .
\end{aligned}
$$

This is done by showing that

$$
\begin{aligned}
\int_{0}^{T}\left\|v_{\varepsilon}\right\|_{L_{\mu}^{5}}^{2} d t \leq & C \int_{0}^{T} \int_{\Omega} a_{i j} \partial_{i} v_{\varepsilon} \partial_{j} v_{\varepsilon} d x d t \\
& +C \int_{0}^{T}\left(\int_{\Gamma} \zeta\left|v_{\varepsilon}\right|^{p+1} d s\right)^{\frac{2}{p+1}} d t+C \int_{0}^{T}\left(\int_{\Lambda \cap \Sigma} G v_{\varepsilon}^{4} v_{\varepsilon} d \mu\right)^{\frac{2}{5}} d t
\end{aligned}
$$

and

$$
\begin{gathered}
\int_{0}^{T} \int_{\Omega 2} a_{i j} \partial_{i} v_{\varepsilon} \partial_{j} v_{\varepsilon} d x d t \leq \varepsilon\left\|v_{\varepsilon}\right\|_{L^{2}\left(L_{\mu}^{5}\right)} f_{\varepsilon}-g_{\varepsilon} \\
\int_{0}^{T}\left(\int_{\Gamma} \zeta\left|v_{\varepsilon}\right|^{p+1} d s\right)^{\frac{2}{p+1}}+\left(\int_{\tilde{\Omega}} G v_{\varepsilon}^{4} v_{\varepsilon} d \mu\right)^{\frac{2}{5}} d t \leq \varepsilon\left\|v_{\varepsilon}\right\|_{L^{2}\left(L_{\mu}^{5}\right)} f_{\varepsilon}+h_{\varepsilon}
\end{gathered}
$$


where $f_{\varepsilon} \rightarrow 0$ and $h_{\varepsilon}-g_{\varepsilon}$ can be ignored for sufficiently small $\varepsilon$.

The derivation of the estimates (7.26) and (7.27) is done as in Sec. 5 except for adding integration over $(0, T)$ to all terms. The major difference is the appearance of the time derivative when deriving (7.26). However, this additional term can be handled in the following manner. First, we integrate by parts

$$
-\int_{0}^{T}\left\langle u_{2}^{\prime}(t)-u_{1}^{\prime}(t), v_{\varepsilon}(t)\right\rangle d t=\int_{0}^{T}\left\langle u_{2}-u_{1}, v_{\varepsilon}^{\prime}\right\rangle d t-\left(u_{2}(T)-u_{1}(T), v_{\varepsilon}(T)\right) .
$$

Since $u_{2}-u_{1}=v_{\varepsilon}$ in $\Omega_{0}(t) \backslash \Omega_{\varepsilon}(t)$ and $v_{\varepsilon}^{\prime}(t)=0$ in the complement of $\Omega_{0}(t) \backslash \Omega_{\varepsilon}(t)$, we have

$$
\int_{0}^{T}\left\langle u_{2}-u_{1}, v_{\varepsilon}^{\prime}\right\rangle d t=\int_{0}^{T}\left\langle v_{\varepsilon}, v_{\varepsilon}^{\prime}\right\rangle d t=\frac{1}{2}\left(v_{\varepsilon}(T), v_{\varepsilon}(T)\right)
$$

and therefore,

$$
-\int_{0}^{T}\left\langle u_{2}^{\prime}-u_{1}^{\prime}, v_{\varepsilon}\right\rangle d t=\left(v_{\varepsilon}(T), \frac{1}{2} v_{\varepsilon}(T)-\left(u_{2}(T)-u_{1}(T)\right)\right) \leq 0 .
$$

Hence, the additional term can be ignored and derivation of the comparison principle can be completed as in the proof of Theorem 4 .

Acknowledgments. The research of the first author was supported by a Grant \# 2785 from the Academy of Finland, Foundation of Emil Aaltonen and COMAS graduate school.

\section{REFERENCES}

[1] Jöran Bergh and Jörgen Löfström, Interpolation Spaces. An Introduction, Grundlehren der Mathematischen Wissenschaften, No. 223, Springer-Verlag, Berlin-New York, 1976

[2] R. A. Bialecki, Solving heat radiation problems using the boundary element method, Computational Mechanics Publications, 1993

[3] Robert Dautray and Jacques-Louis Lions, Mathematical analysis and numerical methods for science and technology, Vol. 3, Spectral Theory and Applications, Springer-Verlag, New York, 1990

[4] M. Delfour, G. Payre, and J. Zolesio, Approximation of nonlinear problems associated with radiating bodies in space, SIAM J. Numer. Anal. 24, 1077-1094 (1987)

[5] Herbert Gajewski, Konrad Gröger, and Klaus Zacharias, Nichtlineare Operatorgleichungen und Operatordifferentialgleichungen, Akademie-Verlag, Berlin, 1974

[6] David Gilbarg and Neil S. Trudinger, Elliptic Partial Differential Equations of Second Order, Springer-Verlag, New York, 1983

[7] G. H. Hardy, J. E. Littlewood, and G. Pólya, Inequalities, Cambridge University Press, 1988

[8] E. Hennebach, P. Junghanns, and G. Vainikko, Weakly singular integral equations with operatorvalued kernels and an application to radiation transfer problems, Integral Equations Operator Theory 22, 37-64 (1995)

[9] C. T. Kelley, Existence and uniqueness of solutions of nonlinear systems of conductive-radiative heat transfer equations, Transport Theory Statist. Phys. 25, 249-260 (1996)

[10] Michal Křížek and Liping Liu, On a comparison principle for a quasilinear elliptic boundary value problem of a nonmonotone type, Applicationes Mathematicae 24, 97-107 (1996)

[11] Mika Laitinen and Timo Tiihonen, Integro-differential equation modelling heat transfer in conducting, radiating and semitransparent materials, Math. Meth. Appl. Sci. 21, 375-392 (1998)

[12] Michael Metzger, Existence for a time-dependent heat equation with non-local radiation terms, Math. Meth. Appl. Sci. 22, 1101-1119 (1999)

[13] M. F. Modest, Radiative Heat Transfer, McGraw-Hill, New York, 1993

[14] Jindřich Nečas, Les méthodes directes en théorie des équations elliptiques, Masson et Cie, Éditeurs, Paris; Academia, Éditeurs, Prague, 1967 
[15] C. Perret and P. Witomski, Équation de la chaleur et réflections multiples, Ann. Inst. Henri Poincaré Anal. Non Linéaire 8, 677-689 (1991)

[16] Rogerio Martins Saldanha da Gama, Existence, uniqueness and construction of the solution of the energy transfer problem in a rigid and nonconvex black body, Z. Angew. Math. Phys. 42, 334-347 (1991)

[17] Rogerio Martins Saldanha da Gama, An alternative mathematical modelling for coupled conduction/radiation energy transfer phenomenon in a system of $N$ gray bodies surrounded by a vacuum, Internat. J. Non-Linear Mechanics 30, 433-447 (1995)

[18] Robert Siegel and John R. Howell, Thermal Radiation Heat Transfer, Hemisphere, third edition, 1992

[19] E. M. Sparrow and R. D. Cess, Radiation Heat Transfer, Hemisphere, augmented edition, 1978

[20] Timo Tiihonen, Stefan-Boltzmann radiation on non-convex surfaces, Math. Meth. Appl. Sci. 20, 47-57 (1997)

[21] Timo Tiihonen, A nonlocal problem arising from heat radiation on non-convex surfaces, European J. Appl. Math. 8, 403-416 (1998)

[22] Lutz Weis, Integral operators and changes of density, Indiana Univ. Math. J. 31, 83-96 (1982)

[23] Eberhard Zeidler, Nonlinear Functional Analysis and its Applications. II/B: Nonlinear Monotone Operators, Springer-Verlag, New York, 1990 